\title{
CAMA
}

Centre for Applied Macroeconomic Analysis

\section{A comment on Wu and Xia (2015), and the case for two-factor Shadow Short Rates}

\section{CAMA Working Paper 48/2015 December 2015}

\section{Leo Krippner}

Reserve Bank of New Zealand and

Centre for Applied Macroeconomic Analysis (CAMA), ANU

\begin{abstract}
Shadow Short Rates (SSRs) estimated from shadow/lower-bound term structure models (SLMs) can be useful for monitoring of the stance of unconventional monetary policy and for quantitative analysis, but only if they are relatively robust. I show from several perspectives that SSRs from three-factor SLMs, which includes Wu and Xia (2015) SSRs, are not robust, and how that arises from the inherent flexibility of three-factor SLMs. Such SSRs should therefore be avoided. However, I also show that estimated SSRs from two-factor SLMs are relatively robust. Hence, two-factor SLM SSRs appear to be good candidates for monitoring and quantitative analysis, but ideally with appropriate robustness checks including alternative monetary policy metrics.
\end{abstract}




\section{Keywords}

Shadow Short Rates, zero lower bound, unconventional monetary policy, term structure models.

\section{JEL Classification}

E43, G12, G13

\section{Address for correspondence:}

(E) cama.admin@anu.edu.au

ISSN 2206-0332

The Centre for Applied Macroeconomic Analysis in the Crawford School of Public Policy has been established to build strong links between professional macroeconomists. It provides a forum for quality macroeconomic research and discussion of policy issues between academia, government and the private sector.

The Crawford School of Public Policy is the Australian National University's public policy school, serving and influencing Australia, Asia and the Pacific through advanced policy research, graduate and executive education, and policy impact. 


\title{
A comment on Wu and Xia (2015), and the case for two-factor Shadow Short Rates
}

\author{
Leo Krippner*
}

16 December 2015

\begin{abstract}
Shadow Short Rates (SSRs) estimated from shadow/lower-bound term structure models (SLMs) can be useful for monitoring of the stance of unconventional monetary policy and for quantitative analysis, but only if they are relatively robust. I show from several perspectives that SSRs from three-factor SLMs, which includes $\mathrm{Wu}$ and Xia (2015) SSRs, are not robust, and how that arises from the inherent flexibility of three-factor SLMs. Such SSRs should therefore be avoided. However, I also show that estimated SSRs from two-factor SLMs are relatively robust. Hence, two-factor SLM SSRs appear to be good candidates for monitoring and quantitative analysis, but ideally with appropriate robustness checks including alternative monetary policy metrics.
\end{abstract}

JEL classification: E43, G12, G13

Keywords: Shadow Short Rates; zero lower bound; unconventional monetary policy; term structure models.

\section{Introduction}

In this article, I show that Shadow Short Rates (SSRs) estimated from three-factor shadow/lower bound term structure models (SLMs) are not robust, explain why they are so sensitive, and illustrate that those issues may be resolved with two-factor SLMs.

As background, SSRs from SLMs have been proposed as a metric for the routine monitoring of the stance of unconventional policy and quantitative analysis; see Krippner (2011-2015) as cited by Bullard (2012, 2013), and Wu and Xia (2015) as cited by Hamilton (2013), Higgins and Meyer (2013), and Zumbrun (2014). ${ }^{1}$ As those authors detail, SSRs can freely take on negative values to reflect a near-zero policy rate plus unconventional policy actions, whereas the policy rate itself would be an uninformative quantity due to its constraint at near-zero values.

*Reserve Bank of New Zealand and Centre for Applied Macroeconomic Analysis. Email: leo.krippner@rbnz.govt.nz. I thank Edda Claus, Iris Claus, Arne Halberstadt, Hibiki Ichiue, and Jing Cynthia $\mathrm{Wu}$ for helpful comments.

${ }^{1}$ Lombardi and Zhu (2014) creates an alternative indicator for the stance of monetary policy, which is also named the SSR, but it is derived from central balance sheet data, monetary aggregates, interest rates, and credit spreads. 
However, SSRs will only be useful for monitoring and quantitative analysis if they are relatively robust, in terms of the profiles and dynamics of SSR point estimates, suitably narrow confidence intervals, and consistency with monetary policy events. Without that relative robustness, the policy interpretation of levels and changes in SSRs and the results of quantitative analysis obtained with them may be tenuous and/or insignificant. In turn, the robustness of SSRs depends on their sensitivity to all aspects of their estimation, including the model specification and the data.

As I will show in section 3, SSRs estimated from three-factor SLMs are not robust. I will illustrate their empirical sensitivity for the United States using the $\mathrm{Wu}$ and Xia (2015, hereafter WX) model, given its prominence from a policy monitoring perspective, ${ }^{2}$ with respect to the choice of lower bound (LB) parameter and different sample periods for estimation. The sensitivity of three-factor SSR estimates to the LB parameter has previously been mentioned and illustrated in Christensen and Rudebusch (2015a),Bauer and Rudebusch (2015), and Krippner (2015c), while Krippner (2013b) discusses other perspectives of empirical sensitivities (e.g. to the maturity span of the yield curve data and to the estimation method). WX acknowledges model-based variation in SSR estimates but downplays its empirical implications, i.e.:

"We confirm that different model choices do influence the level of the shadow rate. However, the common dynamics among different shadow rates point to the same economic conclusion. We also demonstrate that the shadow rate is a powerful tool to summarize useful information at the ZLB."

In fact, the two sets of SSR estimates reported in WX and alternatives mentioned as robustness checks are all obtained from models with relatively high LB parameters, with respect to the data and closely related SLM estimates; e.g. Kim and Priebsch (2013), Krippner (2013c), and Priebsch (2013). I show that adding two alternative and very plausible lower bounds informed by highly relevant market-quoted data obtains SSR point estimates with distinctly different profiles and dynamics to those reported in WX. Furthermore, reestimating the benchmark WX model with expanded samples obtains distinctly different SSR point estimates to those reported in WX and updated monthly by the authors. Finally, the confidence intervals around the benchmark WX SSR point estimates indicate they are insignificantly different from zero even well into the LB period for the United States. These results suggest that the results of the macroeconomic application in WX are quite dependent on the specific modeling choices made by the authors. In addition, WX SSR series are not well correlated with the evolution of unconventional monetary policy events, so their applicability to monitoring levels and changes to the stance of monetary policy is questionable.

Perhaps more important than any specific empirical results are the fundamental reasons why SSR estimates from three-factor SLMs are so sensitive, because the answer suggests that such SSRs will not in general be not suitable for policy monitoring and quantitative analysis. Hence, in section 4 I show that the LB parameter relative to the shorter-maturity data and the inherent flexibility provided by three factors is what leads to very sensitive and sometimes counterintuitive SSRs.

\footnotetext{
${ }^{2}$ For example, the WX SSR series is available from the Federal Reserve Bank of Atlanta (see "https://www.frbatlanta.org/cqer/research/shadow_rate.aspx\#shadow_rate.aspx?panel=1"), and from Haver Analytics.
} 
One ready and straightforward resolution to the issues associated with SSRs from three-factor SLMs is simply to use two-factor SLMs, which I discuss in section 5 . In particular, I show that SSR estimates obtained with different LB parameters and maturity spans of yield curve data maintain very similar profiles and dynamics. Of course, using two factors represents a material constraint relative to three-factor SLMs, and will therefore fit the yield curve data less closely. However, the robustness improvements over threefactor SSRs suggest that the trade-off is worthwhile, assuming one seeks SSR estimates as a straightforward and relatively robust summary monetary policy metric rather than necessarily a closer fit to the data. Ichiue and Ueno (2013) also suggests using SLMs with less than three factors to avoid over-fitting and counterintuitive SSR estimates. In addition, two-factor SSRs closely replicate the information contained in "policy rate liftoff" metrics for both two- or three-factor SLMs.

Two-factor SSRs therefore appear to be good candidates for routinely monitoring of the stance of unconventional monetary policy and for quantitative analysis. ${ }^{3}$ Nevertheless, the magnitudes of negative SSR point estimates from two-factor SLM remain somewhat sensitive between different estimations. Therefore, if two-factor SSR estimates are used as a single monetary policy metric for quantitative analysis over periods spanning non-LB and LB periods, it will be important to test the robustness of those results with a variety of alternative two-factor SSR estimates. In addition, one should ideally also test the robustness with alternative monetary policy metrics, which I mention in the conclusion.

The article follows the order I have already outlined above, with the addition in section 2 of an overview section on SLMs relevant to this article and a discussion of market data relevant to LB parameters for the United States.

\section{Overview of SLMs and the lower bound}

In this section I provide an overview of the key elements of SLMs relevant to this article. Section 2.1 discusses the SLM framework, and section 2.2 discusses the lower bound parameter and plausible range of values applicable to US yield curve data.

\subsection{SLM framework and specification}

The concept of SLMs was originally introduced in Black (1995), and is based on the lower bound mechanism:

$$
\underline{\mathrm{r}}(t)=\max \left[\mathrm{r}(t), \mathrm{r}_{\mathrm{LB}}\right]
$$

where $r(t)$ is the SSR that can freely adopt negative values, and $\underline{r}(t)$ is the lower-bounded or actual short rate which is constrained to a minimum value of the LB parameter $r_{L B}$. Unfortunately, the direct application of the Black (1995) framework with any dynamic process to represent the SSR is relatively intractable, so examples have generally been limited to one or two factor models; e.g. Bomfim (2003), Gorovoi and Linetsky (2004), Ichiue and Ueno (2006), and Kim and Singleton (2012).

Krippner (2011-2015) derives a framework with a Gaussian affine term structure model (GATSM) process for the SSR that closely approximates the Black (1995) framework and is much more tractable, for any number of factors. WX derives the discrete-time

\footnotetext{
${ }^{3}$ Indeed, Francis, Jackson, and Owyang (2014) has already compared Krippner (2011-2015) two-factor SSRs with WX three-factor SSRs in a macroeconomic VAR model, and find better results with the former.
} 
equivalent. ${ }^{4}$ The key result in both derivations is the closed-form analytic expression for lower-bounded forward rates $\underline{\mathrm{f}}\left(x_{t}, \tau\right)$, i.e.:

$$
\underline{\mathrm{f}}\left(x_{t}, \tau\right)=\mathrm{r}_{\mathrm{LB}}+\left[\mathrm{f}\left(x_{t}, \tau\right)-\mathrm{r}_{\mathrm{LB}}\right] \cdot \Phi\left[z\left(x_{t}, \tau\right)\right]+\omega(\tau) \cdot \phi[z(t, \tau)]
$$

with:

$$
z\left(x_{t}, \tau\right)=\frac{\mathrm{f}\left(x_{t}, \tau\right)-\mathrm{r}_{\mathrm{LB}}}{\omega(\tau)}
$$

where $\tau$ is the time to maturity, and $\Phi[\cdot]$ and $\phi[\cdot]$ are respectively the unit normal cumulative density and density functions. The shadow forward rate function $\mathrm{f}\left(x_{t}, \tau\right)$ and volatility function $\omega(\tau)$ are dependent on the model specification in terms of the state variables $x_{t}$ and their associated parameters, which I discuss shortly below.

Equation 2 is the basis for the measurement equation when estimating SLMs, because it provides model results to compare to yield curve data. The data may be forward rates generated from parametrically fitted yield curve data (such as that available using the Gurkaynak, Sack, and Wright (2007, hereafter GSW) data set), or $\underline{\mathrm{f}}\left(x_{t}, \tau\right)$ may be transformed so that the measurement equation can use interest rates or security prices. ${ }^{5}$

The state equation is obtained from the vector Ornstein-Uhlenbeck process that the state variables follow under the physical $\mathbb{P}$ measure, i.e.:

$$
x_{t}=\theta+\kappa\left[\theta-x_{t-1}\right]+\sigma
$$

where $x_{t}$ is the $N \times 1$ vector of state variables, with a long-run value of $\theta$, a meanreversion matrix $\kappa$, and a volatility matrix $\sigma .{ }^{6}$ The linear market price of risk specification $\Pi(t)=\gamma+\Gamma x_{t}$ provides the risk-adjusted $\mathbb{Q}$ measure process for the state variables, which is analogous to equation 4 with $\tilde{\kappa}=\kappa+\Gamma$ and $\tilde{\theta}=\tilde{\kappa}^{-1}(\kappa \theta-\gamma)$. The state variables $x_{t}$ and parameters $\tilde{\kappa}, \tilde{\theta}$, and $\sigma$ define closed-form analytic expressions for $\mathrm{f}\left(x_{t}, \tau\right)$ and $\omega(\tau)$ which, together with the parameter $\mathrm{r}_{\mathrm{LB}}$, define the closed-form analytic expression $\underline{\mathrm{f}}\left(x_{t}, \tau\right)$ in equation 2.

Once the SLM is estimated, with a suitable non-linear Kalman filter, the SSR point estimate is:

$$
\mathrm{r}(t)=a_{0}+b_{0}^{\prime} x_{t}
$$

which is the zero-maturity rate on the estimated shadow forward rate or interest rate curve. As illustrated later throughout this article, SSRs can freely take on negative values. A negative SSR can, in principle, be interpreted as a combination of a near-zero policy rate setting plus unconventional policy actions (e.g. quantitative easing, forward guidance, etc.) that is more accommodative than a near-zero policy rate setting alone.

Specific examples of the framework outlined above are contained in Krippner (20112015) and Christensen and Rudebusch (2013, 2015a,b), with continuous-time specifications, and WX with a discrete-time specification. I will outline some further elements of

\footnotetext{
${ }^{4}$ Christensen and Rudebusch (2015a), Krippner (2015c), and WX show that the approximation is within a maximum of less than 10 basis points for the 10-year maturity. Priebsch (2013) provides an even closer approximation with a second-order method, to within around one basis point, but the framework is more complex and time-consuming to apply.

${ }^{5}$ The zero-coupon transformations are $\underline{\mathrm{R}}\left(x_{t}, \tau\right)=\frac{1}{\tau} \int_{0}^{\tau} \underline{\mathrm{f}}\left(x_{t}, u\right) \mathrm{d} u$ and $\underline{\mathrm{P}}\left(x_{t}, \tau\right)=\exp \left[-\underline{\mathrm{R}}\left(x_{t}, \tau\right) \tau\right]$, and the latter may be used analogously for coupon-paying securities with multiple cashflows; e.g. see Krippner (2015c). Generated instantaneous forward rates may be used to estimate continuous-time versions of the model; there is no need to use generated one-month forward rates as suggested in WX.

${ }^{6}$ All parameter vectors and matrices in this article should be taken as being conformable to $x_{t}$.
} 
those applications in later sections as they relate to this article, but refer readers to the individual sources for the full details.

\subsection{The lower bound for US yield curve data}

Section 2.1 introduces $\mathrm{r}_{\mathrm{LB}}$ as a parameter to be calibrated or estimated when estimating SLBs, in addition to the GATSM state variables and parameters for the shadow term structure. It turns out, as I will detail in sections 3 to 5 , that the LB parameter has a critical influence on the SSR estimates, so I will spend some time discussing it in this section.

A typical calibration for $\mathrm{r}_{\mathrm{LB}}$ in the literature is the theoretically motivated value of zero, because physical currency with an effective nominal interest rate of zero offers a higher yielding substitute investment than any short-maturity deposit with a negative interest rate.

However, it is well-acknowledged that non-zero lower bounds may prevail in practice due to institutional frictions and policy framework choices. ${ }^{7}$ Short-maturity rates and market expectations of their evolutions may therefore be non-zero, and so the SLM should try to appropriately account for those non-zero expectations on the yield curve data.

For the United States, the WX benchmark model uses a calibrated value of 25 basis points (bps $=1 / 100$ percentage points), which is the rate of remuneration on bank reserves held at the Federal Reserve. WX also estimate a similar value of 19 bps, which is close to the $23 \mathrm{bp}$ mean of the shortest maturity generated WX forward rate data (the 1-month rate, three months forward, or $3 \times 1$-month forward rate) from the onset of the LB period up to the end of the WX estimation sample in December $2013 .^{8}$

However, both 25 and 19 bps seem high compared with highly relevant market data available as at end-2013, which I have also illustrated in figure 1 (along with their subsequent updates). Specifically, the minimum (effective) Federal Funds Rate (FFR) since the onset of the LB period to end-2013 was $4 \mathrm{bps}$, with an average of $14 \mathrm{bps}$. The FFR is relevant because it is a benchmark rate for the overnight funding of settlement banks, and hence wholesale financial market rates and rates in the wider economy.

The minimum 3-month Treasury bill (Tbill) rate in the LB period to end-2013 was zero, with an average of 8 bps. The 3 -month Tbill rate is relevant because it is the shortest maturity benchmark interest rate for the government-risk yield curve, which is

\footnotetext{
${ }^{7}$ For example Jarrow (2013) notes that the inconvenience of holding and transacting in physical currency allows materially negative short-maturity interest rates. In practice, many European economies have adopted negative policy rate settings, leading to negative short-maturity interest rates in wholesale financial markets. Alternatively, the United States, Japan, and the United Kingdom have adopted policy frameworks that have led to wholesale short-maturity interest rates remaining mostly non-negative. Note that term interest rates can evolve to negative values due to negative risk premiums (e.g. from safehaven/liquidity demand, etc.) so negative yields on such securities, which have been observed in many markets, does not necessarily indicate a market perception or expectation of a negative lower bound.

${ }^{8}$ I take 16 December 2008 as the unambiguous start of the LB period, because it is the day that the Federal Open Market Committee (FOMC) cut the Federal Funds Rate Target to a range of zero to $25 \mathrm{bps}$ (from the previous level of 1 percent, set on 29 October 2008), and commented that "weak economic conditions are likely to warrant exceptionally low levels of the federal funds rate for some time." Furthermore, the first round of quantitative easing, the so-called QE1, had already been announced on 25 November 2008. Hence, the environment was clearly one of a near-zero policy rate plus both quantitative easing and forward guidance in effect as additional unconventional monetary policy actions. The $25 \mathrm{bp}$ interest rate on both required and excess reserves was also introduced on 16 December 2008.
} 
the curve on which the GSW data set and therefore the generated WX forward rate data are based. In other words, WX use generated forward 1-month government-risk rates, and the 3-month Tbill rate is a market-observed short-maturity rate that offers guidance on the market-perceived LB for government-risk forward rates and interest rates. ${ }^{9}$

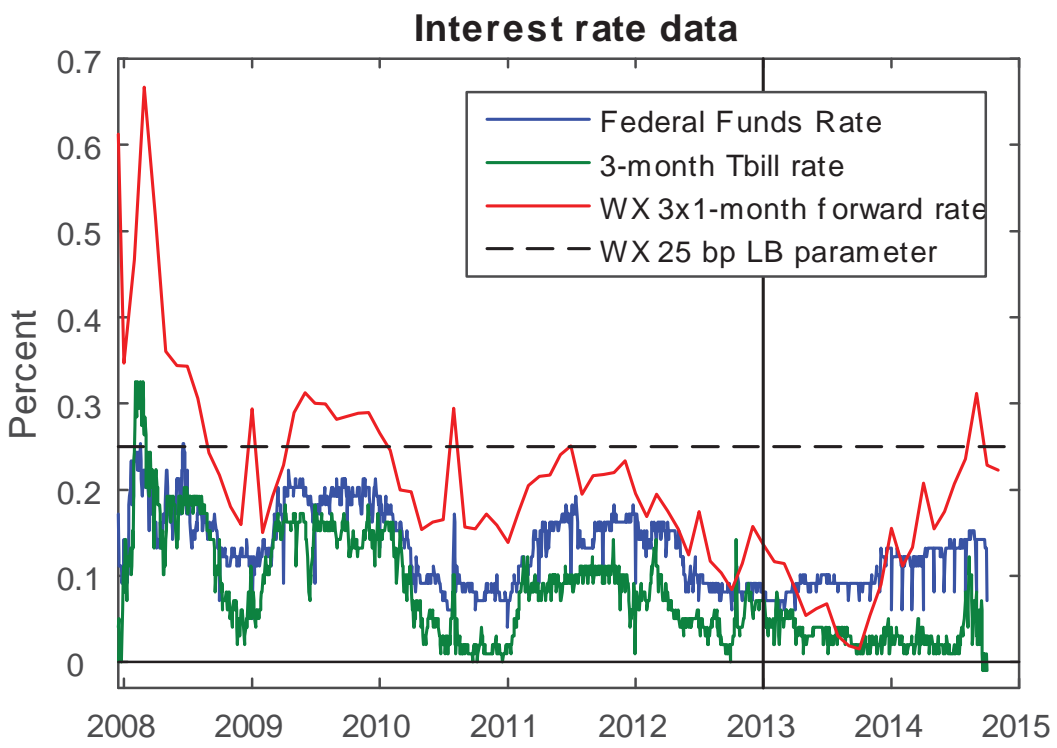

Figure 1: Daily FFR and 3-month Tbill data, and monthly generated WX $3 \times 1$-month forward rates. The FFR and 3-month Tbill minimums and averages suggest plausible lower bounds between zero and $14 \mathrm{bps}$, which are lower than the WX values of 25 and 19 bps.

Hence, even at the time of the original WX analysis to December 2013, it would have been entirely reasonable to test the robustness of the SSR results to LB parameters of less than 25 and 19 bps. In section 3, I use calibrated values of zero and 14 bps to capture the range of minimums and averages mentioned in the previous paragraph, and zero also happens to align with the theoretically motivated LB parameter calibration. The zero to 14 bp range also includes the LB parameters estimated for three-factor SLMs with US data in Kim and Priebsch (2013), Krippner (2013c), and Priebsch (2013), which range from 10 to 14 bps. $^{10}$

\section{Empirical sensitivity of three-factor SSRs}

In this section I empirically demonstrate the sensitivity of three-factor SSRs. I use the WX model to provide specific examples because the WX SSRs are quite prominent and

\footnotetext{
${ }^{9}$ I use the three-month Tbill rate because it is the shortest-maturity Tbill rate from the Federal Reserve Economic Database (FRED) with direct secondary-market quotes. The one-month generated constant maturity Tbill rates from FRED, which have a maturity more relevant to the WX 1-month forward rate data, obtains very similar results; respectively a minimum and mean of zero and 7 bps over the LB period to end-2013.

${ }^{10}$ Of course, there are many alternatives to setting or estimating the LB parameter, including an allowance for time-varying values as in Lemke and Vladu (2015), which may be more realistic as financial markets adapt to updated policy settings and frameworks. However, time-invariant values are sufficient to establish the sensitivity of three-factor SSRs for the United States in this article.
} 
the codes on which they are based have recently been made available, ${ }^{11}$ but I also mention related results available in the literature.

In section 3.1, I replicate the WX SSR point estimates, and then estimate SSRs from the WX SLM using the two LB parameters discussed in section 2.2. I also calculate the confidence intervals associated with all of those WX SSR estimates. I proceed in section 3.2 to estimate the WX model with data beyond 2013. In section 3.3, I discuss the correlation of the WX SSR profiles and changes to the evolution of unconventional monetary policy events.

Note that my focus in this section and the remainder of the article is on the general properties of different classes of SSR estimates as a monetary policy metric. Hence, I will only mention the likely implications of my alternative WX SSR estimates (and later my two-factor SSR estimates) on the WX factor-augmented vector autoregression (FAVAR) application.

\subsection{Results from WX 2013 data set}

In this section I estimate the WX model with WX forward rate data from January 1990 to December 2013, which is the sample used for estimation in WX and the associated working papers from early 2014. I use four different LB specifications. The first two LB specifications replicate the results reported in WX, i.e. a calibrated 25 bp LB parameter for the benchmark model, and an estimated LB parameter of 19 bps for the explicit SSR robustness check. I also estimate the WX model with a calibrated LB parameter of zero and $14 \mathrm{bps}$, based on the discussion in section 2.2. ${ }^{12}$ In all cases, I have included the out-of-sample updates for the SSR estimates beyond December 2013; i.e. I use model parameters estimated up to December 2013 applied to the WX forward rate data set extended to September 2015. The results for the 25 bp LB parameter case exactly match the results reported in the monthly WX updates (to September 2015 at the time of my analysis).

The results are illustrated in figure 1. I have also included indicator arrows for major unconventional monetary policy events, which I list and discuss in section 3.3, and an indicator to mark the end of the WX estimation sample in December 2013.

It is evident that the SSR point estimates from the WX three-factor SLM differ markedly with only minor changes to the LB parameter. The magnitudes differ by up to around two percentage points, and the profiles and dynamics are distinctly different up to mid-2013. In particular, the zero and 14 bp SSR series are flat up to mid-2013, while the 19 and 25 bp series show a downward trend. The zero and 14 bp LB parameter mod-

\footnotetext{
${ }^{11}$ The MatLab code associated with WX can be obtained from the website "http://faculty.chicagobooth.edu/jing.wu/", along with monthly updates of the SSR series from the benchmark WX model. The parameter initialization values I have used when estimating the WX models with a calibrated LB parameter are the parameters from the benchmark WX model. When estimating the WX model with an estimated LB parameter, the parameter initialization values are those from the WX model with an estimated LB parameter. I have also tested many of my WX model estimations with alternative starting values, and have obtained the same results to within numerical tolerances.

${ }^{12}$ Statistically, the log likelihood ratios relative to the estimated LB parameter case rejects all of the calibrated LB parameters, but the 14 bp case is most preferred (i.e. least rejected), followed by the $25 \mathrm{bp}$ and zero bp cases. These results continue to apply for all of the updates in section 3.2, except the $14 \mathrm{bp}$ case is not rejected for the December 2014 update.
} 
els also include materially positive SSR point estimates, which is counterintuitive given the market-observed data in section 2.2 maintained near-zero values throughout the LB period. ${ }^{13}$ I will explain this phenomenon in section 4.

The sensitivities above would have been apparent even at the time of the original WX analysis if a wider range of lower bounds had been tested and reported. In any case, the earlier drafts of Christensen and Rudebusch (2015a), Bauer and Rudebusch (2015), and Krippner (2015c) subsequently provided illustrations of the sensitivity of SSRs estimated from three-factor SLMs, so the point could have been established and reported in subsequent WX revisions.

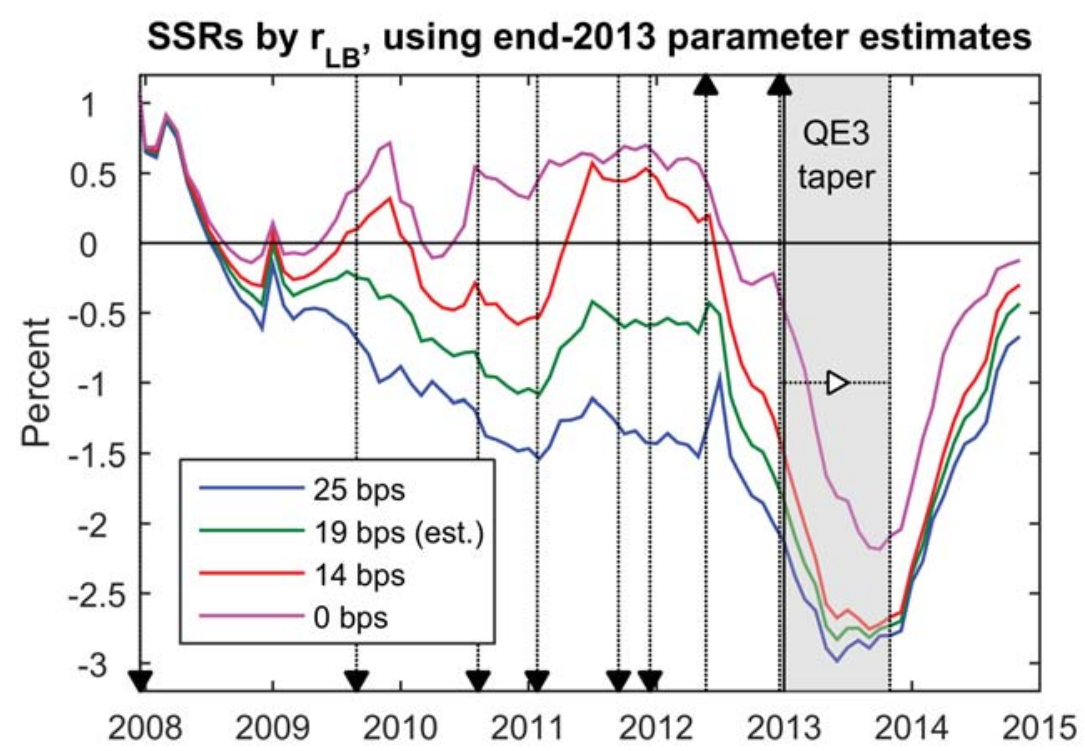

Figure 2: SSR point estimates from WX model estimated with data to December 2013 and using different LB parameters. The magnitudes, profiles, and dynamics of the in-sample and out-of-sample SSRs differ markedly between specifications. The arrows indicate major unconventional policy events, which are listed and discussed in section 3.3.

In addition to the model uncertainty, SSR point estimates will also be subject to estimation uncertainty. WX does not report confidence intervals for the SSR but, given $\mathrm{r}(t)=$ $a_{0}+b_{0}^{\prime} x_{t}$, they can be readily estimated from the standard error $\sigma_{\mathrm{r}(t)}=\sqrt{\sigma_{a_{0}}+b_{0}^{\prime} P_{t} b_{0}}$, where $P_{t}$ is the estimated state variable covariance matrix at time $t$ and $b_{0}=[1,1,0]^{\prime}$ for the WX model. ${ }^{14}$

Figure 3 shows that the confidence intervals around the SSR series are relatively wide compared to the point estimates. Hence, the estimated SSRs are often insignificantly different from zero, with respect to a 95 percent confidence interval. For example, the benchmark WX SSR series only became significantly negative around late-2010, which is approximately two years after the LB period began in the United States. The WX 19 bp

\footnotetext{
${ }^{13}$ The WX SSR estimates for the euro area and the United Kingdom also contain periods of SSRs materially above the relevant policy rate settings and wholesale financial market rates for those economies.

${ }^{14} \mathrm{I}$ use the Hessian from the WX estimation to calculate $\sigma_{a_{0}}$, which obtains the conservative values of $0.34,0.33,0.31$, and 0.28 percentage points, respectively. The robust standard error reported in WX for $a_{0}$ (the WX parameter $\delta_{0}$ in table 1 ) is 1.0551 percentage points, which would make the confidence intervals substantially wider.
} 
SSRs only became significantly negative from when the tapering of QE3 was foreshadowed in mid-2013.
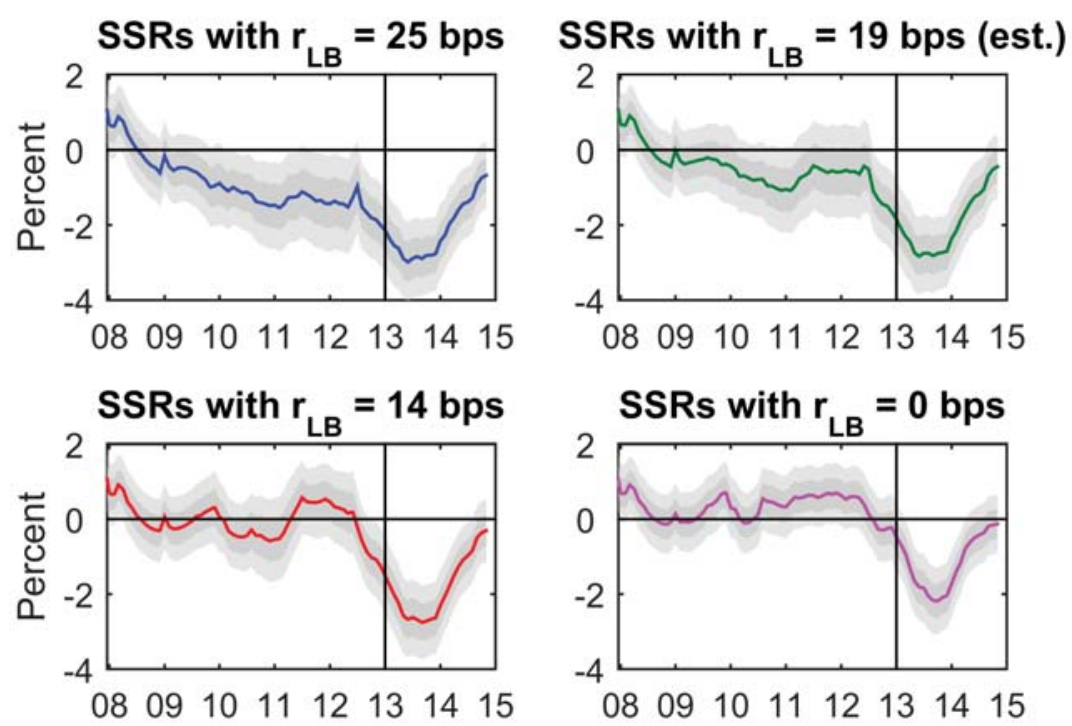

Figure 3: SSR point estimates and confidence intervals ( \pm one standard deviation and 95 percent interval) from the WX model estimated with data to December 2013 and using different LB parameters. The confidence intervals indicate that the SSR estimates are often insignificantly different from zero.

Regarding implications for the WX FAVAR application, the latter results are likely to be quite specific to the estimation choices made by the authors; in particular not testing the SLM estimation with smaller LB parameters or accounting for uncertainty in the SSR estimates. The profile differences of the alternative SSR point estimates are of particular note, because WX comments that "The comovement rather than the difference in levels between the shadow rates is what drives the key results." WX show that the FAVAR results are not significantly affected by using the SSR point estimates from the WX SLM with an estimated LB parameter of 19 bps, which has a correlation of 0.93 relative to the benchmark 25 bp SLM over the LB period to December 2013. However, the correlation drops to 0.54 and 0.14 respectively for $\mathrm{r}_{\mathrm{LB}}=14$ bps and $\mathrm{r}_{\mathrm{LB}}=0$ bps specifications, so the FAVAR results will be less robust to the alternative SSR series produced with only a further minor lowering of the LB parameter. ${ }^{15}$ In addition, by not accounting for the confidence intervals of the estimated SSRs, the confidence intervals for the impulse response functions from the WX FAVAR will be understated.

More generally, if WX SSR point estimates were used for routinely monitoring the level and changes of stance of monetary policy, the interpretations would be very different between different LB parameter specifications. The statistical insignificance of WX SSR point estimates well into the LB period for the United States would also be challenging to interpret.

\footnotetext{
${ }^{15} \mathrm{WX}$ also notes that the FAVAR application is robust to SSR estimates obtained from alternative yield curve data and a two-factor specification. The SLMs used to obtain those alternative SSR estimates use a calibrated LB parameter of 25 bps (confirmed in correspondence with the authors associated with an earlier draft of the present article). Hence, those alternative FAVAR results are again likely to be quite specific to the choice of a relatively high LB parameter.
} 


\subsection{Results from updated WX data sets}

To test how the WX SSR estimates change with updated samples, in this section I reestimate the $25 \mathrm{bp}$ and estimated LB parameter SLMs from WX using data for three alternative sample periods. The first update adds just four extra months of data, ending in April 2014. The second and third alternatives update the sample period to December 2014 and September 2015 respectively.

Figures 4 and 5 illustrate the results, and I have again extended the SSR series associated with each of the reestimates to September 2015. The confidence intervals are those associated with the estimates from the full sample of data as at the time of my analysis, i.e. up to September 2015 (the $\sigma_{a_{0}}$ estimates are 0.64 percentage points for both the 25 bp and estimated LB parameter cases).

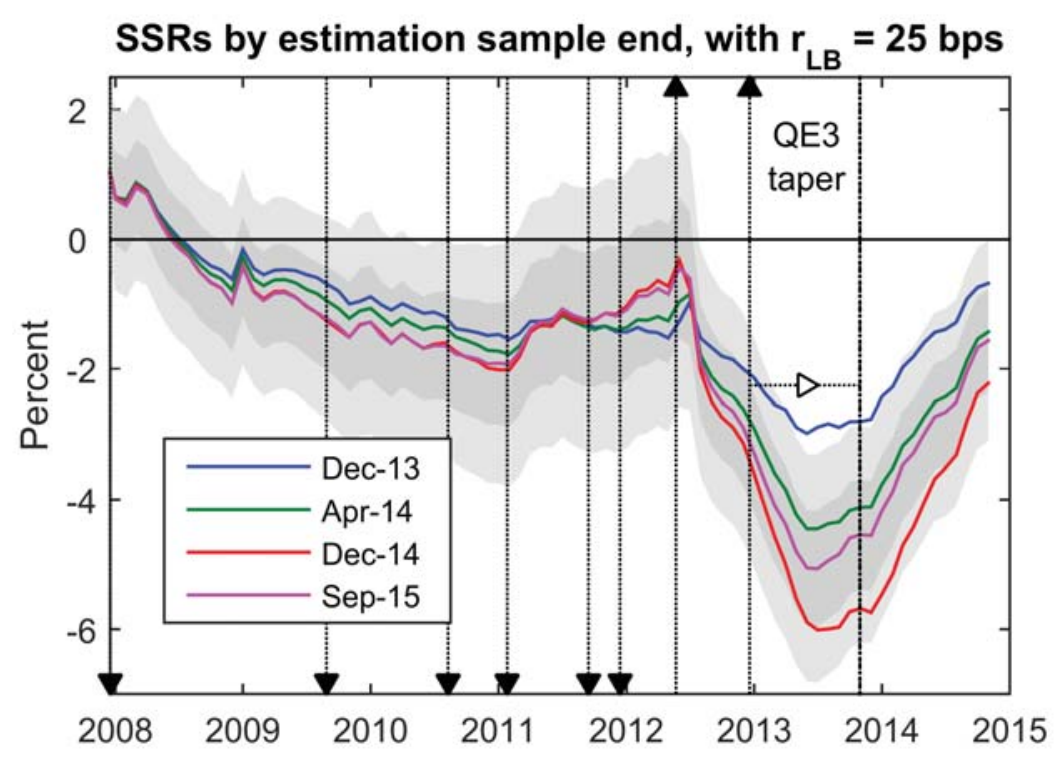

Figure 4: SSR estimates from WX model with a 25 bp LB parameter and reestimations with samples to the given months. Small changes to the sample period results in substantial changes to the later SSR estimates. The arrows indicate major unconventional policy events, which are listed and discussed in section 3.3.

As illustrated in both figures, simply adding four months of data results in a marked change to the benchmark WX SSR estimates. I have chosen April 2014 specifically because it demarks the point at which my month-by-month reestimates of the WX models first showed a distinct change from the estimates to end-2013. However, subsequent monthby-month reestimates and the December 2014 and September 2015 results in figures 4 and 5 show that the changes are persistent. At least the results to April 2014, and likely to December 2014, would have been apparent in WX revisions had the results above been obtained and reported.

A very important corollary to the discussion above is that the regular monthly updates provided by WX continue to use the benchmark WX model and parameters estimated with the sample to December 2013, applied to updated forward rate data. Hence, aside from showing the sensitivity of WX three-factor results, the alternative results illustrated above indicate how the benchmark WX SSR estimates would have changed if the authors had 
occasionally reestimated the model with an updated sample. In particular, the negative values from mid-2012 would have become much more negative.

Like the WX benchmark model, the WX SLM reestimations with an estimated LB parameter (and reestimations with 14 and 0 bp LB parameters, not shown here) also show distinct downside revisions to SSR estimates from mid-2012. In addition, the December 2014 and September 2015 reestimations give SSR estimates with a counterintuitive period of positive values from mid-2012 to mid-2013. These are associated with slightly lower estimates of the LB parameter compared to the December 2013 sample, and I will return to the latter as a source of sensitivity in section 4. Hence, even if the LB parameter was estimated, which avoids any need to justify a particular calibrated value and simply accepts what the yield curve data suggests, the WX SSR estimates would still be sensitive and occasionally counterintuitive.

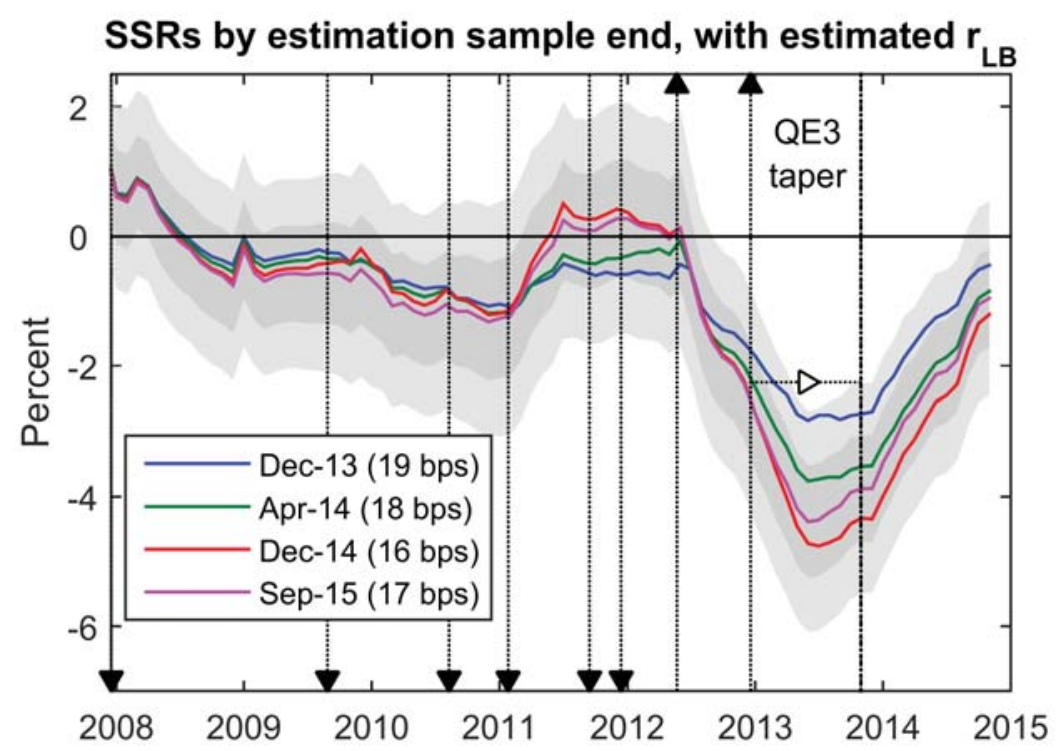

Figure 5: SSR estimates from WX model with an estimated LB parameter and reestimations with data to the given months. Small changes to the sample period results in substantial changes to the later SSR estimates. The arrows indicate major unconventional policy events, which are listed and discussed in section 3.3.

Regarding the WX FAVAR application, if the sample end for the FAVAR remains at December 2013 then it is unlikely that the reestimations above would materially affect the reported WX results. That's because the correlations of the updated series for the LB period to December 2013 remain high; i.e. above 0.85 and 0.80 respectively for the 25 bp and estimated LB parameter cases.

Regarding policy monitoring, using any of the updated SSRs would indicate that the stance of policy became even more accommodative from mid-2013 than the original WX SSR point estimates, and that the prevailing stance of monetary policy (as at the time of my analysis to September 2015) is essentially in line or easier than the stance prior to mid-2013. These results are counterintuitive for the reasons I will detail in the following section. 


\subsection{Consistency with monetary policy events}

Apart from being sensitive, the benchmark WX SSR estimates and the alternatives are also often counterintuitive relative to the evolution of major unconventional policy events. In this section I will focus on the profile and changes to WX SSR estimates; I will discuss the counterintuitive positive SSR estimates separately in the context of section 4 .

The major unconventional policy easing events, which are marked with down arrows where included in figures, are as follows: (1)16 December 2008, FFR target set to a range of zero to 25 bps; (2) 27 August 2010, QE2 foreshadowed;(3) 9 August 2011, calendar forward guidance announced; (4) 21 September 2011, "Operation Twist"; (5) 25 January 2012, calendar forward guidance extended; (6) 13 September 2012, QE3 announced and calendar forward guidance extended; (7) 12 December 2012, QE3 extended and unemployment-based forward guidance announced. The major unconventional policy tightening events, which are marked with up arrows where included in figures, are: (1) 22 May 2013, QE3 tapering foreshadowed; and (2) 18 December 2013, QE3 tapering commenced (the shaded/spanned regions in the figures represents the progressive implementation of tapering until the announced termination of the program on 29 October 2014). Note that the easing event of "Operation Twist" is not shown in the figures, for clarity given its proximity to item 3, but it is included in the correlation analysis below. See Krippner (2015c) for more detail on each of the easing and tightening events discussed in this paragraph.

The first counterintuitive episode for WX SSR point estimates is at the start of the LB period. That is, all WX SSR point estimates only reached negative values in July 2009 and were above 25 bps until April 2009 (inclusive). These dates are well after 16 December 2008 when the FFR target range of zero to 25 bps was set along with associated forward guidance, and QE1 had already been announced (see footnote 8). In other words, a near-zero policy rate plus unconventional monetary policy actions were already in place, but the still-positive WX SSR estimates do not reflect that degree of accommodation.

The second counterintuitive episode is from early-2012 to mid-2013. The SSR estimates for many WX models and/or reestimations, particularly those with an estimated LB parameter, show increasing SSR values which indicates a tightening of unconventional monetary policy. Other WX models and/or reestimations show flat SSR profiles during this period. However, the Federal Reserve extended its forward guidance considerably in January 2012 and introduced a third round of quantitative easing (QE3) plus further forward guidance in September and December 2012. Hence, decreasing SSR estimates would be more consistent with the evolution of events.

The third counterintuitive episode is from mid-2013 to mid-2014. All WX SSR estimates become negative (significantly) from around 2013, and their decreasing values indicate an easing of unconventional monetary policy. However, the Federal Reserve began to remove unconventional monetary policy accommodation during that period, firstly with Chairman Bernanke foreshadowing the tapering of QE3 (in May 2013) and then with the implementation of QE3 tapering (in December 2013, with QE3 ending in October 2014). Note that WX interprets the entire QE3 period as an easing in the stance of policy, and hence consistent with the decline in the benchmark WX SSR. However, the QE3 tapering period amounted to a signal and then a reduction in money supply growth, which are both generally acknowledged to represent a tightening in monetary policy. 
Table 1:

Kendall correlations of major unconventional monetary policy events with WX SSR changes

\begin{tabular}{cccc}
\hline LB parameter & $\Delta$ SSR0 & $\Delta$ SSR 1 & $\Delta$ SSR2 \\
\hline $\mathrm{r}_{\mathrm{LB}}=25$ bps & 0.18 & 0.09 & 0.00 \\
$\mathrm{p}$ value & 0.33 & 0.44 & 0.56 \\
$\mathrm{r}_{\mathrm{LB}}=19$ bps (est.) & 0.09 & 0.09 & -0.53 \\
$\mathrm{p}$ value & 0.44 & 0.44 & 0.97 \\
$\mathrm{r}_{\mathrm{LB}}=14$ bps & 0.09 & -0.45 & -0.62 \\
$\mathrm{p}$ value & 0.44 & 0.94 & 1.00 \\
$\mathrm{r}_{\mathrm{LB}}=0$ bps & -0.45 & -0.45 & -0.62 \\
$\mathrm{p}$ value & 0.94 & 0.94 & 1.00 \\
\hline
\end{tabular}

Note: Major unconventional policy events, as noted in section 3.3, have been assigned values of -1 for easings and +1 for tightenings.

The correlations with WX SSR changes are statistically insignificant.

With such a small number of major unconventional monetary policy events available, it is not possible with typical statistical tests to thoroughly test the relationship between the events and SSR movements. However, the Kendall rank correlation coefficient provides one quantitative indicator. ${ }^{16}$ I have assigned values of -1 and +1 respectively to the easing and tightening events listed earlier. Table 1 then provides the Kendall correlations for those events with changes in estimated SSRs from the month-end prior to the event to three different month-ends after the event. The first is the month-end immediately following the event, which I denote $\Delta \mathrm{SSR} 0 .{ }^{17}$ The others are the subsequent two months, which I denote $\Delta$ SSR1 and $\Delta$ SSR2. I include these to gauge the persistence of the SSR movements with respect to the major unconventional monetary policy events.

The results show that all the correlations are insignificant (and, apart from the 25 bp results, many of the correlation point estimates are negative). In other words, easing (tightening) events are not significantly associated with declines (increases) in the WX SSR point estimates. ${ }^{18}$

\section{Reasons for sensitivity of three-factor SSRs}

In this section, I discuss the reasons why three-factor SLM SSRs are so sensitive, and sometimes also counterintuitive. These reasons are most apparent from the perspective

\footnotetext{
${ }^{16}$ The Kendall rank correlation coefficient is a non-parametric estimate of the association between two quantities based on their concordance. The probabilities are calculated using exact permutation distributions for small samples. See the MatLab function "corr" for further details.

${ }^{17}$ This monthy change is the minimum possible window with the end-month WX SSR estimates, and is sufficient for the purposes of this article (including the comparison to two-factor SSR estimates in section 5). Calculating daily SSR estimates around the events would be interesting and more accurate, because the SSR change would be more clearly identified with the event.

${ }^{18}$ Once again, I have reported the results using the SSRs obtained from parameter estimates up to December 2013, so it is apparent that the correlations between the estimated SSR changes and the events could have been obtained and reported at that time (all of the events are prior to December 2013). Using reestimated WX SSRs with updated samples obtains very similar results.
} 
of their cross-sectional properties, i.e. how the estimated three-factor SLMs represent the yield curve data at each point in time.

Hence, in section 4.1, I first present and discuss the WX shadow and lower-bounded forward rate curves, and related information, that are the basis for obtaining the WX SLM model results and SSRs in section 3. I then illustrate and discuss a particular example of the WX SLM cross-sectional results to explain why the WX forward rate factors in combination with small LB parameter specification changes can lead to marked changes in estimated SSRs. In section 4.3, I discuss why the issues with WX SSR estimates will be present for all three-factor SLMs estimated from three-factor SLMs.

\subsection{WX results}

The WX SLM shadow forward rate curve $\mathrm{f}\left(x_{t}, \tau\right)$ at any point in time $t$ is:

$$
\mathrm{f}\left(x_{t}, j\right)=x_{1, t} \cdot\left[\rho_{1}^{\mathbb{Q}}\right]^{j}+x_{2, t} \cdot\left[\rho_{2}^{\mathbb{Q}}\right]^{j}+x_{3, t} \cdot j\left[\rho_{2}^{\mathbb{Q}}\right]^{j}+\mathrm{VE}(j)
$$

where $x_{1, t}, x_{2, t}$, and $x_{3, t}$ are the state variables at time $t, \rho_{1}^{\mathbb{Q}}$ and $\rho_{2}^{\mathbb{Q}}$ are the two parameters that define the risk-adjusted mean-reversion matrix $\tilde{\kappa}, j$ is the time to maturity $\tau$ expressed in months, and $\operatorname{VE}(j)$ is a relatively minor and time-invariant term that I will simply abbreviate as the "volatility effect" (because it allows for the effect that volatility in the SSR has on expected returns from the shadow yield curve). The SSR estimate is $\mathrm{r}_{t}=a_{0}+x_{1, t}+x_{2, t}$, because $b_{0}=[1,1,0]^{\prime}$ for the WX model.

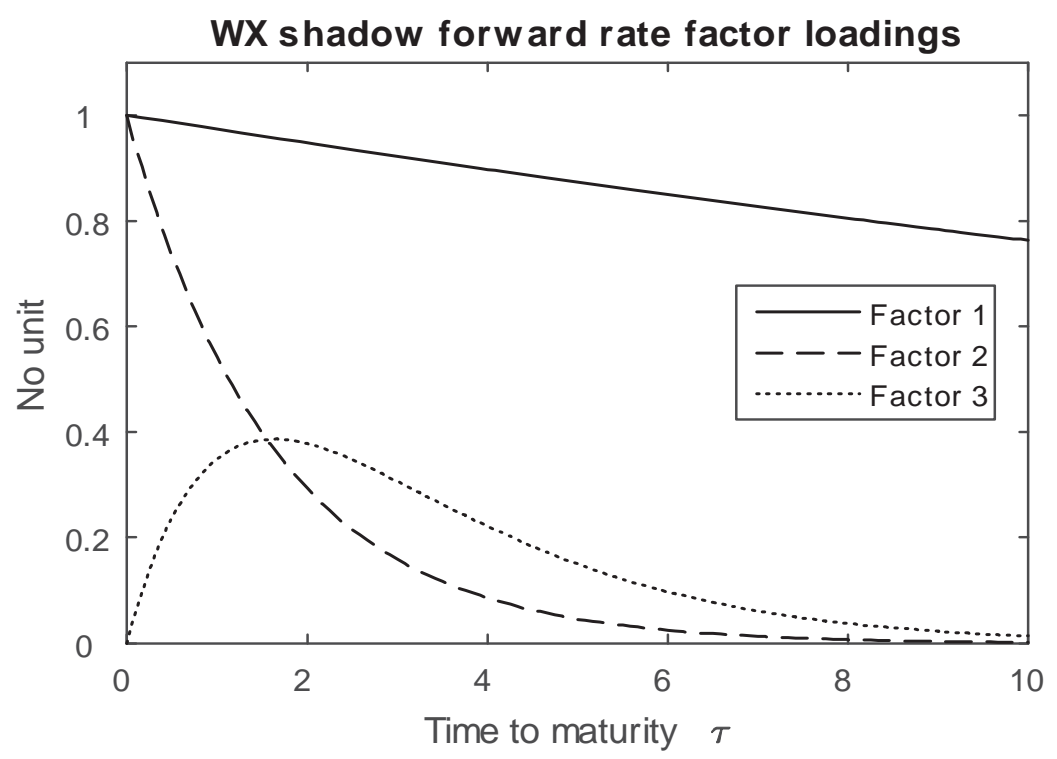

Figure 6: The three WX shadow forward rate factor loadings, which are each associated respectively with the three WX state variables $x_{1, t}, x_{2, t}$, and $x_{3, t}$.

The functions $\left[\rho_{1}^{\mathbb{Q}}\right]^{j},\left[\rho_{2}^{\mathbb{Q}}\right]^{j}$, and $j\left[\rho_{2}^{\mathbb{Q}}\right]^{j}$ are the three factor loadings for the WX SLM shadow forward rate curve, each associated with their respective state variables. Figure 6 plots those three shadow forward rate factors for the benchmark WX model. ${ }^{19}$ Note that

\footnotetext{
${ }^{19}$ I have scaled the WX Factor 3 by $\tilde{\phi} / 12$ in figure 5 , where $\tilde{\phi}=-12 \log \left(\rho_{2}^{\mathbb{Q}}\right)$ with $\rho_{2}^{\mathbb{Q}}=0.9502$ for the benchmark WX model. This scaling simply emphasizes the close relationship of the WX factors to the Level, Slope, and Bow factors discussed in sections 4.2 and 5, and has no implications for the discussion in this section.
} 
the value of the shadow forward rate factors at zero time to maturity is $b_{0}=[1,1,0]^{\prime}$, and also that the shadow forward rate factors for the other three WX models introduced in section 3 are very similar.

The associated WX lower-bounded forward rate function $\underline{\mathrm{f}}\left(x_{t}, \tau\right)$ is obtained by substituting the expression for $\mathrm{f}\left(x_{t}, j\right)$ in equation 6 into equation 2 . The result in full is:

$$
\begin{aligned}
\underline{\mathrm{f}}\left(x_{t}, j\right)= & \mathrm{r}_{\mathrm{LB}} \cdot\{1-\Phi[z(t, j)]\}+\omega(j) \cdot \phi[z(t, j)]+\Phi[z(t, j)] \cdot \operatorname{VE}(j) \\
& +x_{1, t} \cdot\left[\rho_{1}^{\mathbb{Q}}\right]^{j} \Phi[z(t, j)]+x_{2, t} \cdot\left[\rho_{2}^{\mathbb{Q}}\right]^{j} \Phi[z(t, j)] \\
& +x_{3, t} \cdot j\left[\rho_{2}^{\mathbb{Q}}\right]^{j} \Phi[z(t, j)]
\end{aligned}
$$

The last two lines of equation 7 are most important in what follows, while the first line contains all of the other components. In particular, the last two lines contain the state variables multiplied by their respective lower-bounded forward rate factor loadings, where the latter are the shadow forward rate factor loadings attenuated by the cumulative normal distribution function $\Phi\left[z\left(x_{t}, j\right)\right] .{ }^{20}$ Note from equation 3 that $z\left(x_{t}, j\right)=$ $\left[\mathrm{f}\left(x_{t}, j\right)-\mathrm{r}_{\mathrm{LB}}\right] / \omega(j)$, which imparts three effects: (1) equation 7 is a non-linear function of the state variables (because $\Phi[\cdot]$ is a non-linear function); (2) the lower-bounded forward rate factor loadings will vary over time as the state variables themselves vary; and (3) $\mathrm{r}_{\mathrm{LB}}$ relative to $\mathrm{f}\left(x_{t}, \tau\right)$ will influence the lower-bounded forward rate factor loadings.

In practice, the state variables and model parameters are estimated by maximum likelihood using a suitable non-linear Kalman filter. This process estimates the SLM parameters (except $r_{L B}$ if it is calibrated) that apply to the entire yield curve sample, while simultaneously estimating the state variables at each time $t$ that obtain the minimum least squares residual fit to the yield curve data at time $t$, i.e.:

$$
\underline{\mathrm{f}}(t, j)=\underline{\mathrm{f}}\left(x_{t}, j\right)+\eta_{t, j}
$$

where $\underline{\mathrm{f}}(t, j)$ is the forward rate data, $\underline{\mathrm{f}}\left(x_{t}, j\right)$ is the non-linear function of the three state variables $x_{1, t}, x_{2, t}$, an $x_{3, t}$ as given in equation 7 , and $\eta_{t, j}$ are the residuals from the estimation. One key parameter associated with the estimation is $\sigma_{\eta}$, which is the standard error of the residuals, where $\eta_{t, j} \sim \mathrm{N}\left(0, \sigma_{\eta}\right)$. Hence, $\sigma_{\eta}$ is an indicator of the goodness of fit of the model to the data, but it also plays another role as discussed further below. The values of $\sigma_{\eta}$ are very similar in all the estimated WX SLMs from section 3, ranging from 8.7 to 8.9 bps.

All of the elements mentioned above come together in complex way to produce the empirical sensitivity demonstrated in section 3 . Therefore it is helpful to narrate a detailed cross-sectional example before making general comments. I choose November 2012 for this exposition, but similar examples could also be generated at many other points in time. ${ }^{21}$

Figure 7 plots the WX data as at November 2012, along with the LB parameters, estimated LB forward rate functions $\underline{\mathrm{f}}\left(x_{t}, \tau\right)$, and the shadow forward rate functions $\mathrm{f}\left(x_{t}, \tau\right)$

\footnotetext{
${ }^{20} \underline{\mathrm{f}}\left(x_{t}, j\right)$ can be converted to an explicit linearized function of $x_{1, t}, x_{2, t}$, an $x_{3, t}$, which is the basis for the extended Kalman filter estimation in WX, and analogously the iterated extended Kalman filter estimation in Krippner (2015c). However, the intermediate expression for $\underline{\mathrm{f}}\left(x_{t}, j\right)$ here deliberately maintains the lower-bounded forward rate factor loadings, which is useful for the exposition in this section (and later in section 5).

${ }^{21}$ From figure 2, November 2012 is when the greatest range exists between the alternative SSR estimates, which best helps to illustrate the points in this section. However, figure 2 also shows that the wide range of SSR estimates is persistent before and after November 2012, so any other choice around that month would produce similar results.
} 
for the four WX models introduced in section 3. Figure 8 plots the four lower-bounded forward rate factor loadings associated with those estimated models.

Beginning with the $\mathrm{r}_{\mathrm{LB}}=25$ bps case, note that the 0.25-, 0.5-, and 1-year forward rate data are below the LB parameter. Hence, the estimated residuals will at least be equal to the difference between the LB parameter and those data points. More precisely, the near-zero short-maturity values for the $\mathrm{r}_{\mathrm{LB}}=25$ bps lower-bounded factor loadings in figure 8 show that, regardless of the estimated values of the state variables, the SLM cannot improve the short-maturity residuals much beyond the LB parameter/data point gaps. In essence then, the model ignores such data for that yield curve observation and focuses on obtaining a closer fit to the remaining data that are above the LB parameter. In particular, the second state variable adopts a more negative value (than the subsequent cases below) to fit the general slope evident in the longer-maturity data; in this case $x_{2, t}=-1.88 \%$. Given that $\mathrm{r}_{t}=a_{0}+x_{1, t}+x_{2, t}$, the more negative value of $x_{2, t}$ produces a more negative SSR point estimate, $\mathrm{r}_{t}=-1.42 \% .{ }^{22}$

With the slightly lower LB parameter of $\mathrm{r}_{\mathrm{LB}}=19 \mathrm{bps}$, the 0.25-, 0.5-, and 1-year forward rate data are now slightly above the LB parameter. The SLM can now better attempt to fit the shorter-maturity data in addition to the longer-maturity data (i.e. figure 8 shows that the short-maturity values for the lower-bounded factor loadings are slightly larger than for the $25 \mathrm{bp}$ LB parameter case). The model therefore adopts a less negative $x_{2, t}$ state variable estimate, $x_{2, t}=-1.03 \%$, to fit the more moderate slope of the data across the entire yield curve. The less negative value of $x_{2, t}$ in turn produces a less negative SSR point estimate, $\mathrm{r}_{t}=-0.59 \%$.

With the LB parameter slightly lower again, i.e. $\mathrm{r}_{\mathrm{LB}}=14 \mathrm{bps}$, the $0.25-, 0.5-$, and 1-year forward rate data are now above the LB parameter to a material extent relative to the standard error of the residuals $\sigma_{\eta}$; i.e. 9.4, 5.9, and 5.4 bps respectively with $\sigma_{\eta}=8.7$ bps. Those data points, particularly the 0.25 -year forward rate, therefore have relatively more influence on the SLM estimation than the 25 and 19 bp LB parameter cases. In particular, the flexibility of the three-factor WX SLM and the attempt to fit those shortermaturity data points results in a positive $x_{2, t}$ state variable estimate, $x_{2, t}=0.08 \%$, and a positive SSR point estimate, $\mathrm{r}_{t}=0.53 \%$. Figure 8 shows that the inverted bow shape in the lower-bounded forward rate curve arises from the lower-bounded factor loadings 1 and 2 , which in turn reflects two effects: (1) the positive values of $\mathrm{f}\left(x_{t}, \tau\right)-\mathrm{r}_{\mathrm{LB}}$ for near-zeromaturities allow the lower-bounded factor loadings 1 and 2 to adopt the near-one values of the respective shadow factor loadings; ${ }^{23}$ and (2) the negative values of $\mathrm{f}\left(x_{t}, \tau\right)-\mathrm{r}_{\mathrm{LB}}$ for short- to -mid-maturities still greatly attenuates, via $\Phi[z(t, \tau)]$, the short- to midmaturity values of the shadow forward rate loadings 1 and 2 , as for the 25 and $19 \mathrm{LB}$ parameter cases.

The case with the LB parameter at zero extends the $\mathrm{r}_{\mathrm{LB}}=14$ bps result even further. The 0.25-, 0.5-, and 1-year forward rate data are 23.4, 19.9, and 19.4 bps respectively with $\sigma_{\eta}=8.9 \mathrm{bps}$, so their influence relative to longer-maturity data increases further, the $x_{2, t}$ state variable estimate becomes more positive, $x_{2, t}=0.34 \%$, and the SSR point estimate becomes more positive, $\mathrm{r}_{t}=0.70 \%$.

\footnotetext{
${ }^{22}$ The contribution of $a_{0}+x_{1, t}$ and its changes to the SSR estimates and changes are not very material relative to the $x_{2, t}$ state variable, which is why I focus on the latter. The values of $a_{0}+x_{1, t}$ for the four cases are respectively $0.46 \%, 0.44 \%, 0.45 \%$, and $0.36 \%$.

${ }^{23}$ For $\tau=0, \lim _{\tau \rightarrow 0} \Phi\left\{z\left(x_{t}, \tau\right)\right\}=\Phi\left\{\left[\mathrm{r}_{t}-\mathrm{r}_{\mathrm{LB}}\right] / 0\right\}=\Phi\{+\infty\}=1$ when $\mathrm{r}_{t}>\mathrm{r}_{\mathrm{LB}}$. Hence, the values of the lower-bound factor loadings at $\tau=0$ are $[1,1,0]^{\prime} \cdot \lim _{\tau \rightarrow 0} \Phi\left[z\left(x_{t}, \tau\right)\right]=[1,1,0]^{\prime}$.
} 

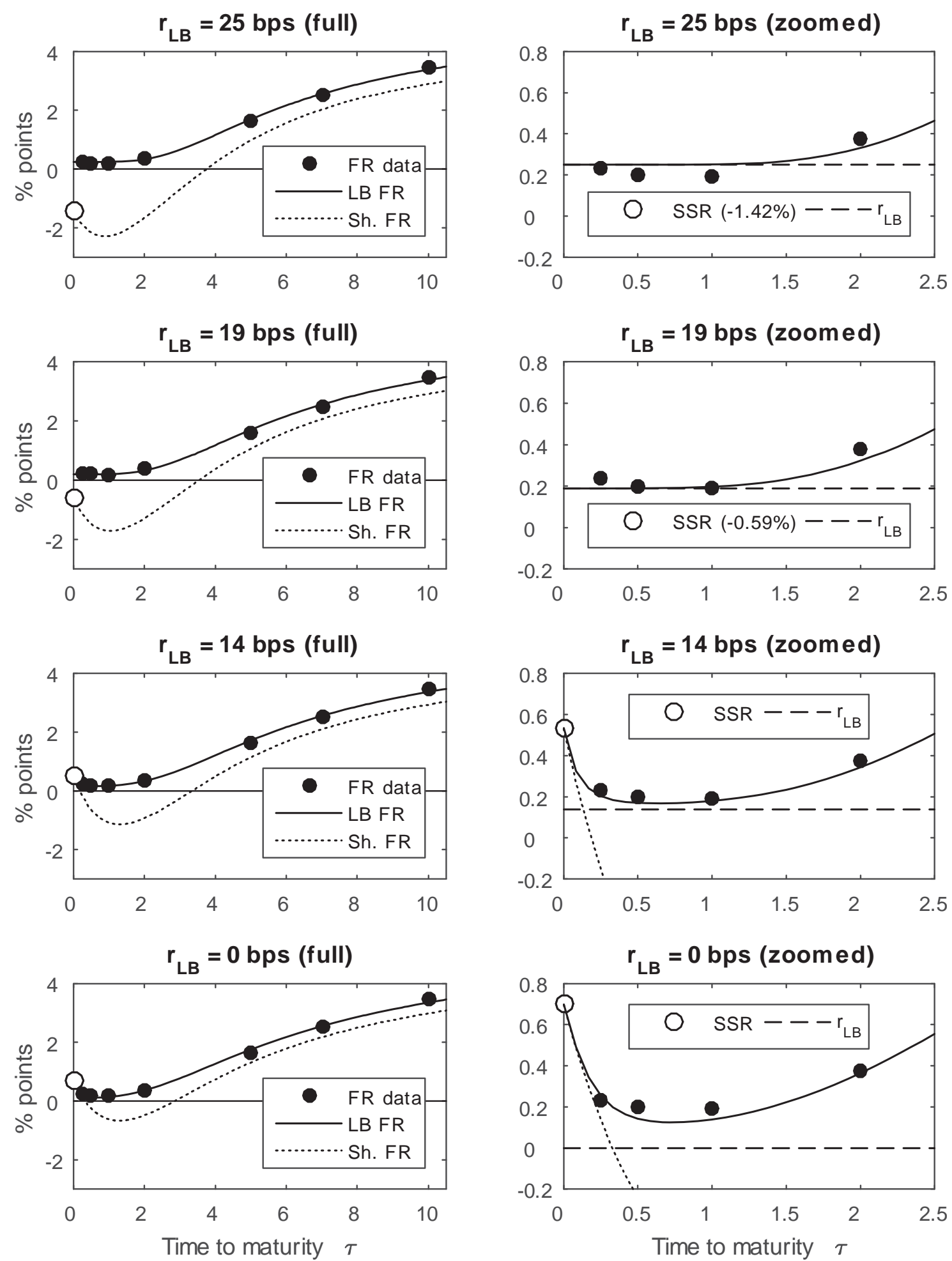

Figure 7: Generated WX forward rate data (FR data), and WX model-estimated lower bound and shadow forward rate curves (LB FR and Sh. FR) for November 2012. As discussed fully in

the text: (1) the non-linearity and flexibility of the three-factor WX SLM makes SSR

estimates highly sensitive to the proximity of short-maturity data relative to the LB parameter; and (2) related to the previous point, short-maturity data materially above the LB can result in counterintuitive positive SSRs in LB periods. 

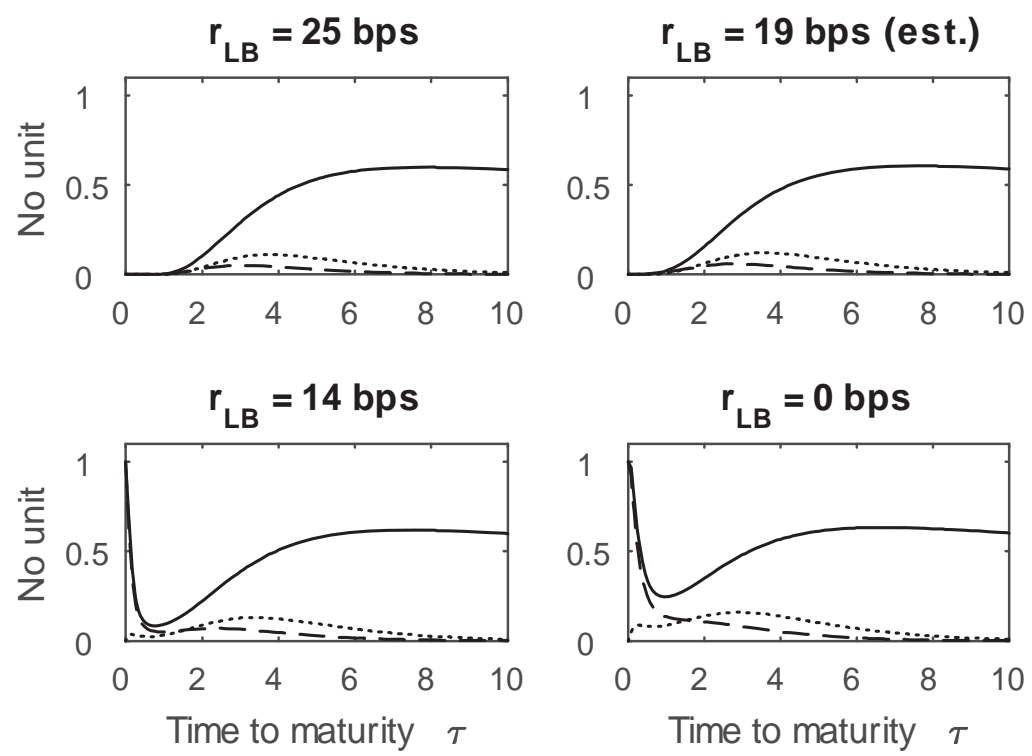

Figure 8: WX lower-bounded forward rate factor loadings for November 2012.

The general point from the discussion above is that small changes in the LB parameter relative to the shorter-maturity data can have a large influence on SSR point estimates due to the non-linearity and flexibility of three-factor SLMs. In essence, shorter-maturity data more below the LB parameter will result in greater effective downweighting of the influence of that data relative to longer maturity data, as indicated in the 25 and 19 bp lowerbounded factor loadings in figure 8 . In turn, the model adopts more negative estimates for the $x_{2, t}$ state variable to better fit the steeper slope in the longer-maturity data, which therefore results in more negative SSR point estimates. ${ }^{24}$ At a point of materiality dependent on the order of magnitude of the SLM goodness of fit to the data, the gap of shorter-maturity data above the LB parameter will influence the model to produce counterintuitive SSR point estimates, i.e. above the LB parameter. This phenomenon occurs even in the WX model with an estimated LB parameter when that model is reestimated with data to December 2014 and September 2014 (see figure 5).

\subsection{The general sensitivity of SSRs from three-factor SLMs}

The results in the previous section are particular to the WX three-factor SLM specification, but this section explains that such results hold in general for SSRs obtained from three-factor SLMs.

Empirically, Bauer and Rudebusch (2015) illustrates the sensitivity of SSRs, with respect to calibrated LB parameters, using a three-factor SLM with an unrestricted discretetime three-factor GATSM specification to represent the shadow term structure (initially within the Black (1995) framework and subsequently within the Priebsch (2013) approximation framework). Progressively altering the LB parameter from zero to $25 \mathrm{bps}$ moves the SSR estimates through a range of up to around 3.5 percentage points, the zero case has counterintuitive positive values during the LB period, and the profiles are distinctly different between the zero and 25 bp cases.

\footnotetext{
${ }^{24}$ The wider confidence intervals associated with the higher LB parameters from figure 3, particularly in the most LB-constrained period, are also consistent with the downweighting reducing the effective amount of data in the cross-sectional estimation.
} 
Krippner (2015c) obtains similar empirical results using the continuous-time threefactor (Level, Slope, and Bow) arbitrage-free Nelson and Siegel (1987) model (ANSM) from Krippner (2006) and Christensen, Diebold, and Rudebusch (2011) to represent the shadow term structure within the Krippner (2011-2015) approximation framework. With three values of the LB parameter (zero, $25 \mathrm{bps,}$, and an estimated $14 \mathrm{bps}$ ) the SSR estimates during the LB period vary by up to around 8 percentage points, ${ }^{25}$ the zero and $14 \mathrm{bp}$ cases have counterintuitive positive values, and all of the profiles are distinctly different.

The three-factor ANSM has the following functional form:

$$
\mathrm{f}\left(x_{t}, \tau\right)=L_{t}+S_{t} \cdot \exp (-\tilde{\phi} \tau)+B_{t} \cdot \tilde{\phi} \tau \exp (-\tilde{\phi} \tau)+\mathrm{VE}(\tau)
$$

where $\tilde{\phi}$ is the single parameter that defines the risk-adjusted mean-reversion matrix $\tilde{\kappa}$. Note that the three-factor ANSM is very similar to the WX shadow term structure specification in equation 6, except there is a constraint of no mean reversion for Factor 1 , so it becomes a constant of $1 .{ }^{26}$ Another constraint is $a_{0}=0$, so the SSR is $\mathrm{r}_{t}=L_{t}+S_{t}$ (given $b_{0}=[1,1,0]^{\prime}$ ). Similar to the discussion in section 4.1, Krippner (2015c) provides a detailed example of how the LB parameter relative to the shorter-maturity data can greatly influence the estimated Slope state variable $S_{t}$ to produce very sensitive SSR estimates. ${ }^{27}$

The Krippner (2015c) results based on the three-factor ANSM also establish that the sensitivity of estimated SSRs will also hold in general for SLMs that use GATSMs with three (or more) factors. The reason is that a generic GATSM with an arbitrary $N \geq 3$ factors can be closely approximated by an ANSM with three factors. This result is established theoretically in Krippner (2015b,c), and Krippner (2015b) shows that the result typically holds empirically when comparing three-factor GATSMs to three-factor ANSMs.

The empirical examples and discussion up to this point have all used and assumed three-factor SLMs estimated from only yield curve data and without any constraints. One potential solution to the sensitive and counterintuitive SSR estimates would therefore be to impose some form of constraints to ensure that the flexibility of three-factor SLMs does not produce "unreasonable" results. For example, related to the previous section, one could constrain the shorter-maturity data or the way the model treats it during LB periods, or constrain the state variable estimates directly. However, depending on how

\footnotetext{
${ }^{25}$ The larger range of magnitudes compared to Bauer and Rudebusch (2015) and the results in this article is due to the use of yield curve data out to 30 years of maturity in Krippner (2015c). The maturity span of the data is another source of empirical sensitivity, as illustrated in Krippner (2013b) and section 5.3 .

${ }^{26}$ That is, $\left[\rho_{1}^{\mathbb{Q}}\right]^{j}=1$ when $\rho_{1}^{\mathbb{Q}}=1$. The other factor loadings relate as follows: $\left[\rho_{2}^{\mathbb{Q}}\right]^{j}=\exp (-\tilde{\phi} \tau)$ and $\frac{\tilde{\phi}}{12} j\left[\rho_{2}^{\mathbb{Q}}\right]^{j}=\tilde{\phi} \tau \exp (-\tilde{\phi} \tau)$, with $\tilde{\phi}=-12 \log \left(\rho_{2}^{\mathbb{Q}}\right)$ and $\tau=j / 12$.

${ }^{27}$ The MatLab code associated with the Krippner (2015c) three-factor SLM can be obtained from the website. "http://www.rbnz.govt.nz/research_and_publications/research_programme/ additional_research/matlab_code.html". Christensen and Rudebusch (2015a) also derives the equivalent three-factor SLM, and shows the sensitivity of SSR estimates to using one, two, and three factors, and mentioned but does not specifically investigate the sensitivity of SSR estimates to the LB parameter. Background material and $\mathrm{R}$ code related to Christensen and Rudebusch (2015a) are available at "http://cepr.org/event/1854".
} 
such constraints are imposed, estimations could become somewhat more challenging and time-consuming.

But one very straightforward and consistent constraint is simply to freely estimate a more parsimonious SLM. This is the topic of the following section. Note that one might argue that setting the LB parameter to a suitably high value so that it appropriately downweights the shorter-maturity data during LB periods is also a straightforward constraint that obtains reasonable SSR estimates. However, this resolution risks being inconsistent with highly relevant financial market data (as discussed for the United States in section 2.2) or the estimated LB parameters (as illustrated in figure 5). In turn, without sound empirical justification, choosing a particular LB parameter to obtain reasonable SSR estimates would be a somewhat subjective exercise.

\section{The case for SSRs from two-factor SLMs}

In this section, I make the case for SSRs estimated from two-factor SLMs. Section 5.1 outlines the specific model and data I will use, and in section 5.2 I repeat the applications from sections 3 and 4 with the two-factor SLM. Section 5.3 provides results with longermaturity data, and section 5.4 discusses the use of two-factor SSR estimates for policy monitoring and quantitative analysis.

\subsection{K-ANSM(2) SLM and data for estimation}

The model I will use to illustrate the case for SSRs from two-factor SLMs is the K$\operatorname{ANSM}(2) .{ }^{28}$ The name is the abbreviation for the continuous-time Krippner (2011-2015) SLM framework of equation 2 using the two-factor (Level and Slope) ANSM to represent the shadow term structure. The shadow forward rate expression is therefore:

$$
\mathrm{f}\left(x_{t}, \tau\right)=L_{t}+S_{t} \cdot \exp (-\tilde{\phi} \tau)+\mathrm{VE}(\tau)
$$

and the SSR is $\mathrm{r}_{t}=L_{t}+S_{t}$ (given $\left.b_{0}=[1,1]^{\prime}\right)$. The associated lower-bounded forward rate function $\underline{\mathrm{f}}\left(x_{t}, \tau\right)$ is:

$$
\begin{aligned}
\underline{\mathrm{f}}\left(x_{t}, \tau\right)= & \mathrm{r}_{\mathrm{LB}} \cdot\{1-\Phi[z(t, \tau)]\}+\omega(\tau) \cdot \phi[z(t, \tau)]+\Phi[z(t, \tau)] \cdot \operatorname{VE}(j) \\
& +L_{t} \cdot \Phi[z(t, j)]+S_{t} \cdot \exp (-\tilde{\phi} \tau) \Phi[z(t, j)]
\end{aligned}
$$

The main reason for using the K-ANSM(2) is because Krippner (2015b,c) show that the two-factor ANSM provides the most parsimonious approximation to a generic GATSM with an arbitrary $N \geq 2$ factors. The K-ANSM(2) SSR results shown in section 5.2 will therefore apply generally regardless of the "true" GATSM process for the shadow yield curve, and they will also be representative of results that could have been obtained by applying SLMs with alternative two-factor specifications for the shadow yield curve. As

\footnotetext{
${ }^{28}$ The model is available on the website "http://www.rbnz.govt.nz/research_and_publications/ research_programme/additional_research/matlab_code.html". Monthly updates of the SSR from the K-ANSM(2) with an estimated LB parameter are available on the website "http://www.rbnz.govt.nz/research_and_publications/research_programme/additional_research/ 5655249.html".
} 
such, it is possible to infer the performance of SSRs estimated from two-factor SLMs in general.

For estimating the $\mathrm{K}-\operatorname{ANSM}(2)$ in section 5.2, I use the interest rate data set described in Krippner (2015c) but with three changes to align more closely to the WX SLM estimations: (1) the data are the 3- and 6-month secondary market Tbill data from FRED, transformed to a continuously compounding zero-coupon basis, and the continuously compounding GSW zero-coupon interest rates for 1, 2, 3, 5, 7, and 10 years (Krippner (2015b) uses that data set, but Overnight Indexed Swap (OIS) rates from January 2006); (2) the 10-year rate is the longest maturity (Krippner (2015b) includes 30-year data); and (3) the measurement equation residuals are specified to be homoskedastic (Krippner (2015b) allows for heteroskedastic measurement equation residuals). ${ }^{29}$ In section 5.3, I estimate the $\mathrm{K}-\operatorname{ANSM}(2)$ as above, but including the 30-year GSW interest rates.

\section{$5.2 \quad \mathrm{~K}-\mathrm{ANSM}(2)$ results with 10-year data}

In this section, I repeat all of the analysis undertaken for the WX three-factor SLM using the $\mathrm{K}-\operatorname{ANSM}(2)$.

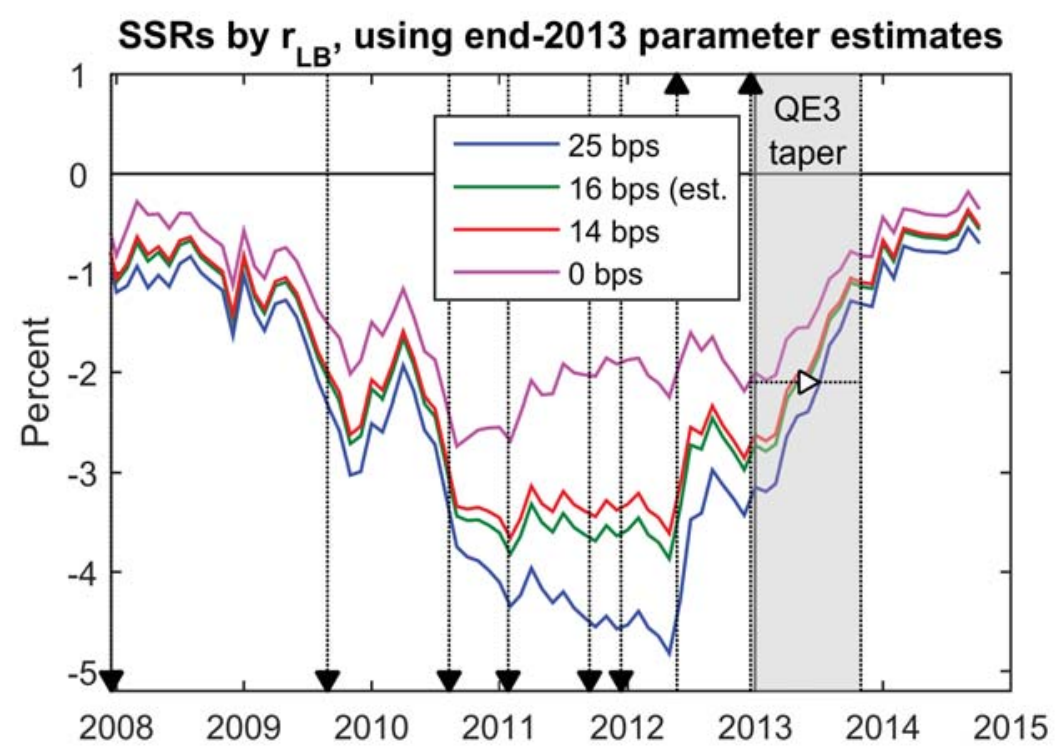

Figure 9: SSRs from K-ANSM(2) estimated with data to December 2013 and using different

LB parameters. The profiles and dynamics of the in-sample and out-of-sample SSRs are all similar, although the magnitudes have some sensitivity. The arrows indicate major unconventional policy events, which are listed and discussed in section 3.3.

Regarding the sensitivity to different LB parameter specifications, figure 9 plots the in- and out-of-sample SSRs estimated from the K-ANSM(2) using data to end-2013 and four different LB parameters, i.e. the calibrated values of 25 bps, 14 bps, and zero bps,

\footnotetext{
${ }^{29}$ The homoskedastic specification offers improvements to the Krippner (2015c) specification from three perspectives: (1) estimation time is shorter, because only a single measurement error parameter estimate is required for the data of all maturities, whereas the heteroskedastic specification requires a parameter for each time to maturity; (2) shorter-maturity and longer-maturity data are fitted more closely; and (3) related to the previous point, the resulting SSR estimates are less volatile and closer to the shortestmaturity rate in non-LB periods. Bauer and Rudebusch (2015) also use a homoskedastic specification.
} 
and an estimated LB parameter of 16 bps. ${ }^{30}$ The point estimates for each SSR series have no counterintuitive non-negative values, and each has similar profiles and dynamics (the correlations between all series are greater than 0.90). However, the magnitudes still have some sensitivity to the LB parameter, with differences in the SSR series of up to around 2.5 percentage points. Bauer and Rudebusch (2015) and Krippner (2015c) have also reported the magnitude sensitivity for SSR estimates from two-factor SLMs. I will discuss it subsequently in the context of figure 12, and sections 5.3 and 5.4 contain related comments.

Figure 10 shows that all of the estimated SSR series are negative to a statistically significantly extent throughout the LB period. The 95 percent confidence intervals are actually very similar to the WX confidence intervals from figure 3 ( $15 \mathrm{bps}$ wider for the 25 bp case, and then 12, 4, and 16 bps narrower for the remaining respective cases), but the K-ANSM(2) SSR point estimates are more negative than the WX SSR point estimates.
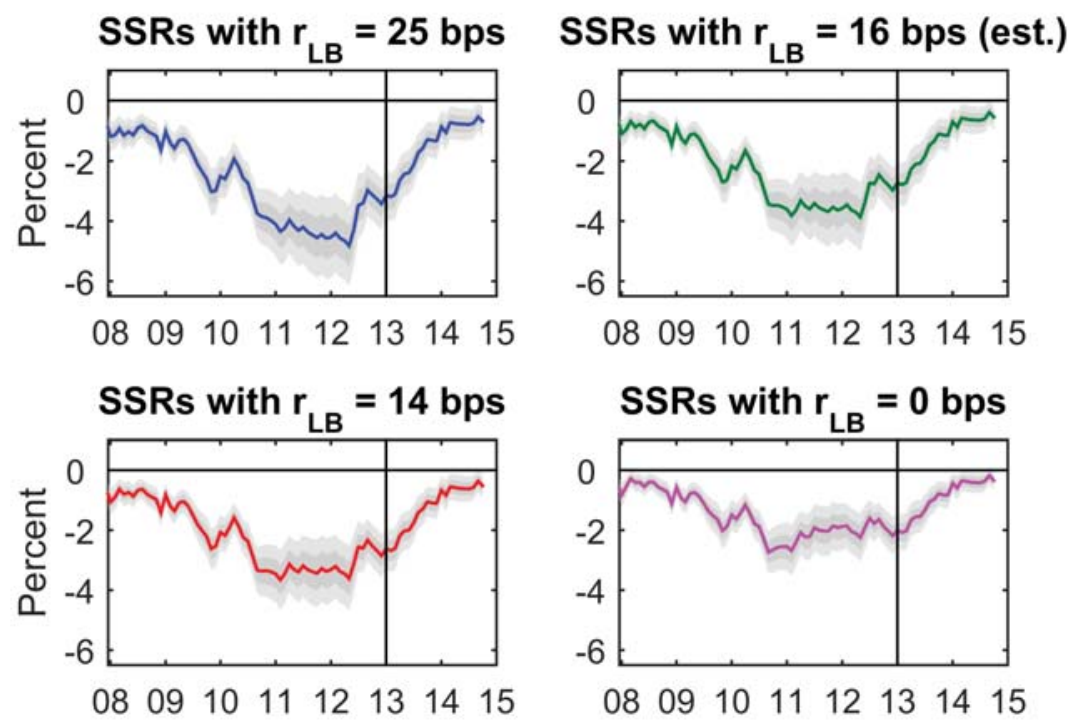

Figure 10: SSR point estimates and confidence intervals ( \pm one standard deviation and 95 percent interval) from K-ANSM(2) model estimated with data to December 2013 and using different lower bounds. The confidence intervals indicate that all SSR estimates are significantly negative.

Regarding the sensitivity to the sample period, figure 11 shows the SSR results from the $\mathrm{K}-\operatorname{ANSM}(2)$ estimated with the same sample periods as the WX results in figure 4. These are the results associated with estimated LB parameters, which is the Krippner (2015c) benchmark model, but the results from all of the models with calibrated LB parameters are similarly stable. The confidence intervals are those associated with the estimates from the full sample of data as at the time of writing, i.e. up to September 2015. Hence, if one had estimated an SSR series from the K-ANSM(2) in December 2013, the history and updates of the SSR series would essentially be the same for subsequent reestimations.

\footnotetext{
${ }^{30}$ Statistically, the $25 \mathrm{bp}$ and zero bp LB parameters are rejected, but the $14 \mathrm{bp}$ case is not. These results continue to apply for all of the updates in this section.
} 


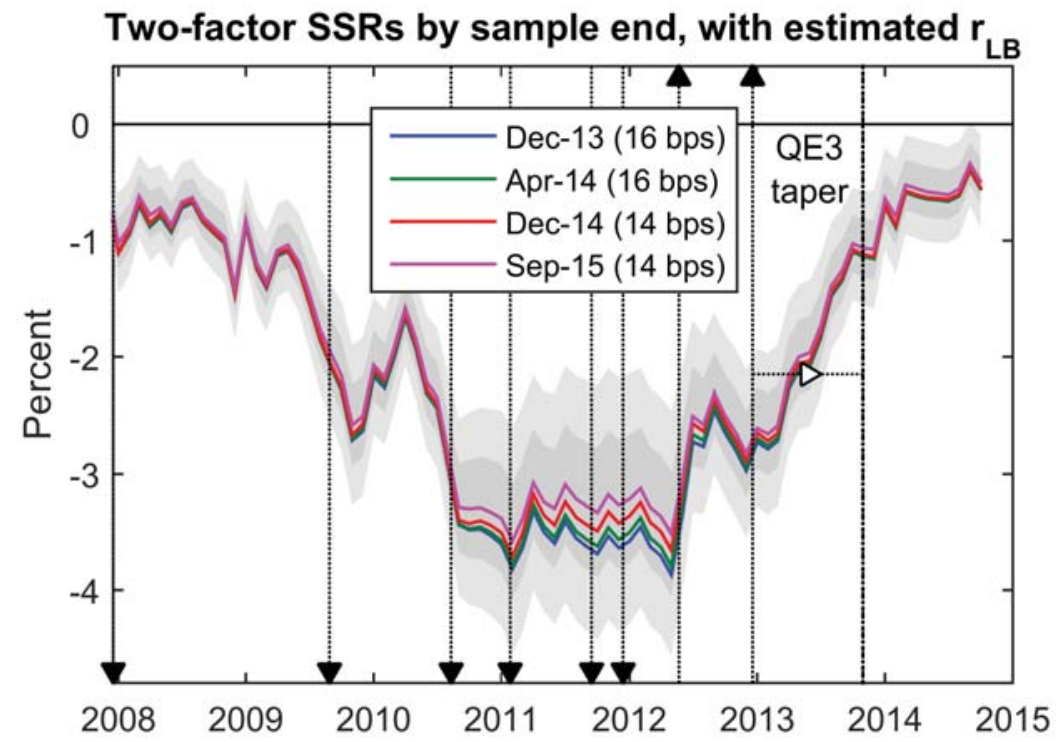

Figure 11: SSRs from K-ANSM(2) with estimated LB parameter and reestimations with data to the given months. Changes to the sample period leave the SSR estimates essentially unchanged. The arrows indicate major unconventional policy events, which are listed and discussed in section 3.3.

Regarding consistency with the evolution of major unconventional monetary events, the K-ANSM(2) SSR point estimates from all four models are very plausible, generally decreasing persistently and/or staying low in response to easing events, and increasing persistently in response to tightening events. In particular, the K-ANSM(2) SSR estimates move consistently with the three episodes discussed in section 3.3, where the WX SSR estimates were counterintuitive. First, all of the K-ANSM(2) SSR point estimates (and 95 percent confidence intervals) are negative at the beginning of the LB period. ${ }^{31}$ Second, with the exception of the zero bp LB parameter case, all of the K-ANSM(2) SSR estimates remain low or decrease from January 2012 to April 2013, which is consistent with the easing events over that period. Third, all of the K-ANSM(2) SSR series increase sharply on Chairman Bernanke's May 2013 foreshadowing of QE3 tapering, and increase steadily as QE3 tapering was progressively implemented from December 2013 to October 2014.

There are two distinct countermovements in the K-ANSM(2) SSR profiles between the major unconventional monetary policy events, but these are readily explainable from the perspective of events at the time. The first countermovement is the SSR increase from late-2010 to early 2011. This increase was associated with a positive run of economic data and market-perceived effectiveness of QE2. Specifically, FFR futures at the time indicated a central market expectation that the Federal Reserve could raise the FFR target beyond 25 bps within around six months. The second countermovement is the SSR increase between late- and end-2013. This decrease occurred in the wake of Chairman Bernanke's QE3 tapering comments as FOMC members sought to assure markets that any tapering

\footnotetext{
${ }^{31}$ The first instance of negative values for all SSR estimates was in November 2008. That consistently reflects the low values of the FFR relative to the FFR target of 1 percent at the time (the November end-month, monthly average, and monthly minimums were respectively $0.53,0.39$, and 0.23 percent), the QE1 announcement on 25 November 2008, and an expectation of a cut to the FFR target in the forthcoming 16 December 2008 FOMC meeting.
} 
would be gradual, and that any subsequent normalization of monetary policy would be some way off. The start of tapering was also subsequently delayed in the midst of debate on the US budget that led to a temporary government shutdown.

The Kendall rank correlation coefficient results contained in table 2 quantitatively confirm, to the extent possible with the limited number of observations, that changes to K-ANSM(2) estimated SSRs are generally consistent with major unconventional policy announcements. That is, all of the Kendall correlation estimates are positive, with eight significant to the 5 percent level of significance, and only two outside the 10 percent level of significance (i.e. for the $\Delta \mathrm{SSR} 1$ and $\Delta \mathrm{SSR} 2$ results with $\mathrm{r}_{\mathrm{LB}}=0$ bps).

Table 2:

Kendall correlations of major unconventional monetary policy events with K-ANSM(2) SSR changes

\begin{tabular}{cccc}
\hline LB parameter & $\Delta$ SSR0 & $\Delta$ SSR 1 & $\Delta$ SSR2 \\
\hline $\mathrm{r}_{\mathrm{LB}}=25$ bps & $0.62^{* *}$ & $0.62^{* *}$ & $0.62^{* *}$ \\
p value & 0.03 & 0.03 & 0.03 \\
$\mathrm{r}_{\mathrm{LB}}=16$ bps (est.) & $0.62^{* *}$ & $0.62^{* *}$ & $0.53^{*}$ \\
$\mathrm{p}$ value & 0.03 & 0.03 & 0.06 \\
$\mathrm{r}_{\mathrm{LB}}=14$ bps & $0.62^{* *}$ & $0.62^{* *}$ & $0.53^{*}$ \\
$\mathrm{p}$ value & 0.03 & 0.03 & 0.06 \\
$\mathrm{r}_{\mathrm{LB}}=0$ bps & $0.62^{* *}$ & 0.36 & 0.45 \\
$\mathrm{p}$ value & 0.03 & 0.17 & 0.11 \\
\hline
\end{tabular}

Note: Major unconventional policy events, as noted in section 3.3, have been assigned values of -1 for easings and +1 for tightenings. The correlations with K-ANSM(2) SSR changes are all positive and most are significant.

Regarding the sensitivity of the K-ANSM(2) SSRs, the cross-sectional results for November 2012 in figure 12 show that even K-ANSM(2) SSR estimates continue to show material variation in magnitude with small changes to the LB parameter. The reasons are closely related to the discussion in section 4 . Specifically, when the LB parameter is more above or less below the shorter-maturity yield curve data, the $\operatorname{K-ANSM}(2)$ will effectively increasingly downweight that data (in the sense described in section 4.2), fit the longer-maturity data with a more negative Slope state variable estimate $S_{t}$, and therefore produce a more negative SSR estimate (given $\left.\mathrm{r}_{t}=L_{t}+S_{t}\right){ }^{32}$

\footnotetext{
${ }^{32}$ The standard error of the residuals $\sigma_{\eta}$ for the four $\mathrm{K}-\operatorname{ANSM}(2)$ estimates are all very similar at either 14 or 15 bps, which accords with the two-factor SLM results in Bauer and Rudebusch (2015), and also shows that the K-ANSM(2) does not fit the yield curve data as closely as three-factor SLMs. Note also that higher LB parameters again produce wider confidence intervals, as figure 10 shows, because the downweighting of the shorter-maturity data reduces the effective amount of data in the cross-sectional estimation.
} 

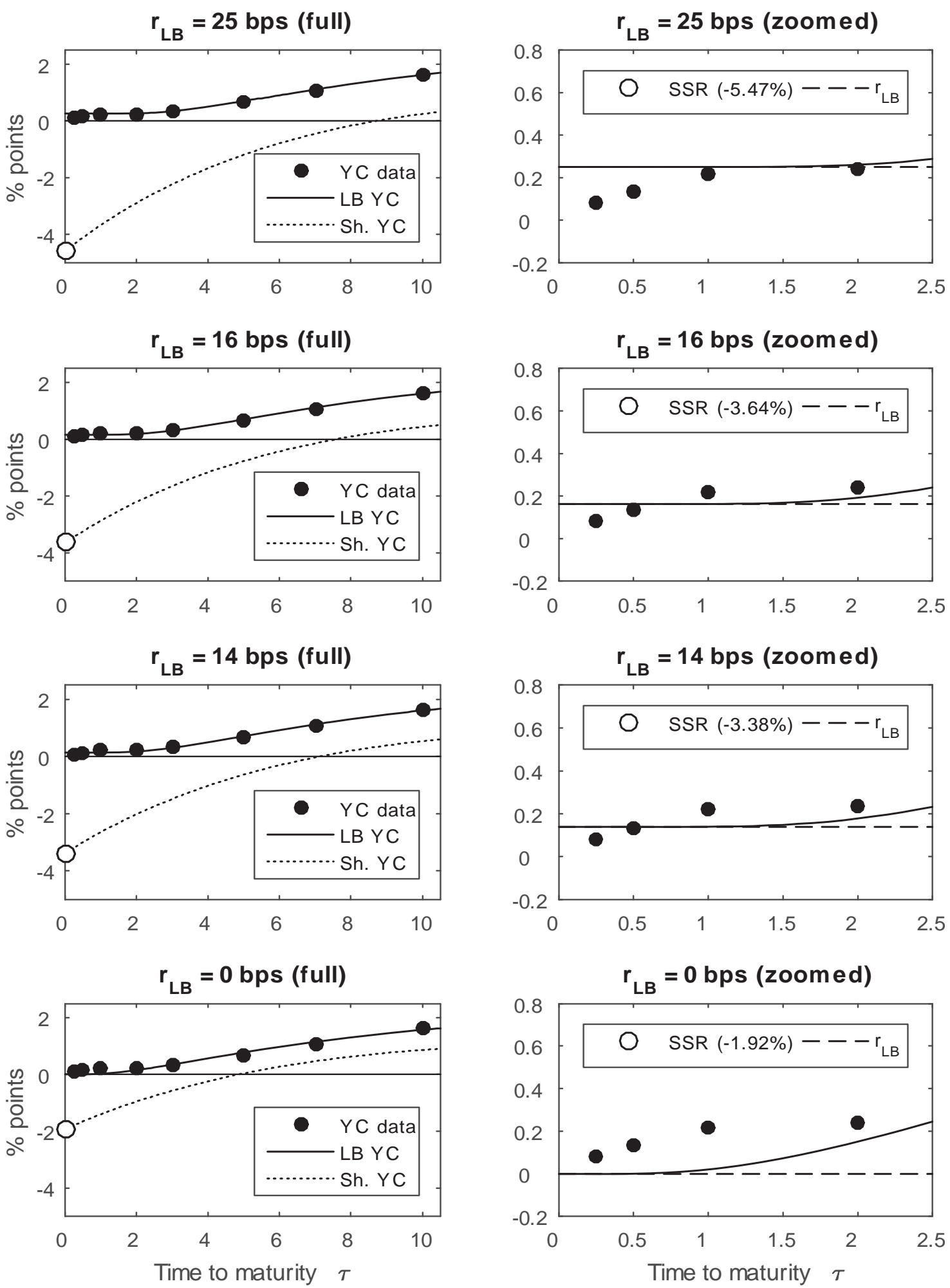

Figure 12: Yield curve data (YC data), and K-ANSM(2) estimated lower bound and shadow forward rate curves (LB YC and Sh. YC) for November 2012. As discussed fully in the text:

(1) there are no instances of counterintuitive positive SSRs; and (2) the estimated SSR remains somewhat sensitive to the proximity of data relative to the LB parameter. 
Figure 13 illustrates the increasing effective downweighting of the shorter-maturity data as the LB parameter is raised relative to that data. ${ }^{33}$ I illustrate another source of magnitude sensitivity in section 5.3, and discuss its general implications for empirical applications in section 5.4.
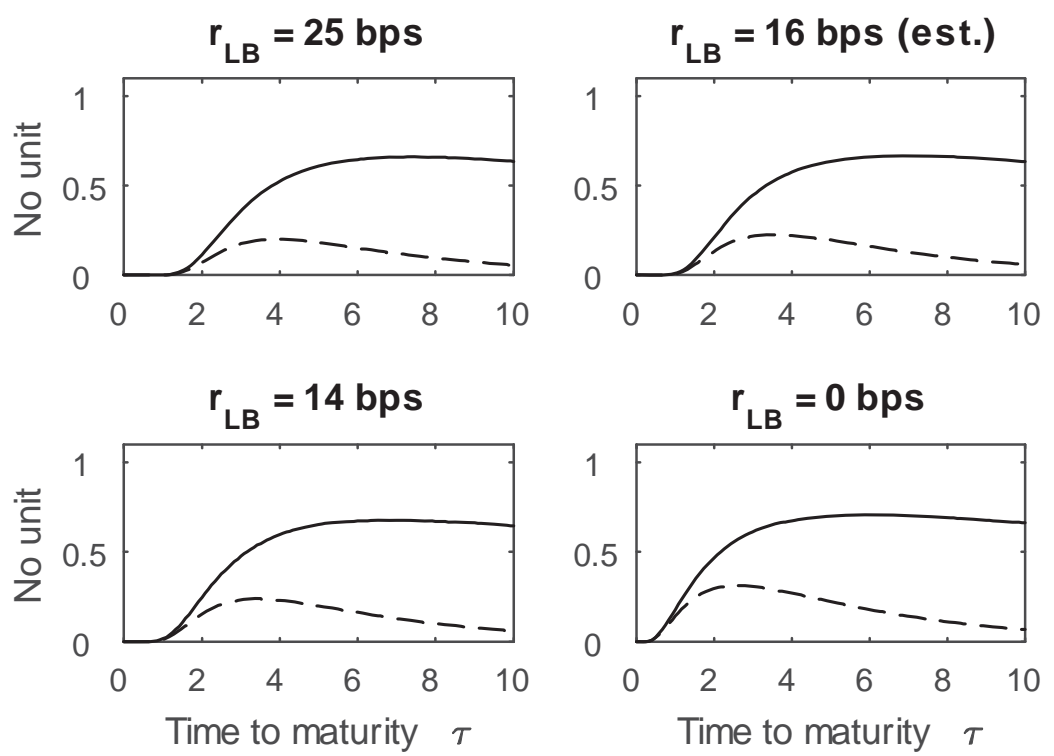

Figure 13: K-ANSM(2) lower-bounded forward rate factor loadings for November 2012.

Despite their magnitude sensitivity, the K-ANSM(2) results in figure 12 have no counterintuitive positive SSR values, and figures 9 to 11 confirm that no such instances occur. It is technically possible to generate positive $\operatorname{SSR}$ with the $\mathrm{K}-\operatorname{ANSM}(2)$ during the LB period, but only artificially using values of short-maturity data well above the LB parameter and mid-maturity data. Specifically, as one of a series of robustness checks on the sensitivity of the K-ANSM(2) SSR estimates for a given LB parameter, I raised the values of all the shorter-maturity data (i.e. 0.25-, 0.5, and 1-years over the entire sample) while leaving the longer-maturity data unchanged. Generating counterintuitive positive SSR values for November 2012 with the zero bp LB specification (the most sensitive K$\operatorname{ANSM}(2)$, due to the lower effective downweighting as discussed above) required raising the shorter-maturity data by 50 bps (resulting in level for November 2012 above the 2and 5-year data, and similar to the 7-year data). Smaller changes to the shorter-maturity data (even up to $45 \mathrm{bps}$ ) and larger changes to only part of the sample left the magnitudes, profiles, and dynamics of the SSR results similar to the original estimates. Importantly, these results indicate that the magnitudes (for a given LB parameter specification), profiles, and dynamics of K-ANSM(2) SSR estimates for a given LB parameter will be robust to alternative sources of interest rate data for the estimation, and even to material errors in the data.

\footnotetext{
${ }^{33}$ I have plotted K-ANSM(2) lower-bounded forward rate factors for comparability with figure 7. Lowerbounded interest rate factors are actually used in the $\mathrm{K}-\operatorname{ANSM}(2)$ estimation given that the yield curve data are interest rates. The forward rate and interest rate factor loadings both contain the same downweighting effect, but simply from different perspectives.
} 


\subsection{K-ANSM(2) results with 30-year data}

As a further robustness check for two-factor SSR estimates, I have reestimated the K$\operatorname{ANSM}(2)$ with an estimated LB parameter and an extended maturity span of yield curve data, i.e. adding 30-year data as used in Krippner (2011-2015). ${ }^{34}$ This maturity span is also the basis for the SSR estimates from Krippner (2015c) that are updated monthly and reported on the website "http://www.rbnz.govt.nz/research_and_ publications/research_programme/additional_research/5655249.html", ${ }^{35}$ so it is worthwhile checking the properties of SSR estimates with an extended maturity span against those reported in section 5.2.

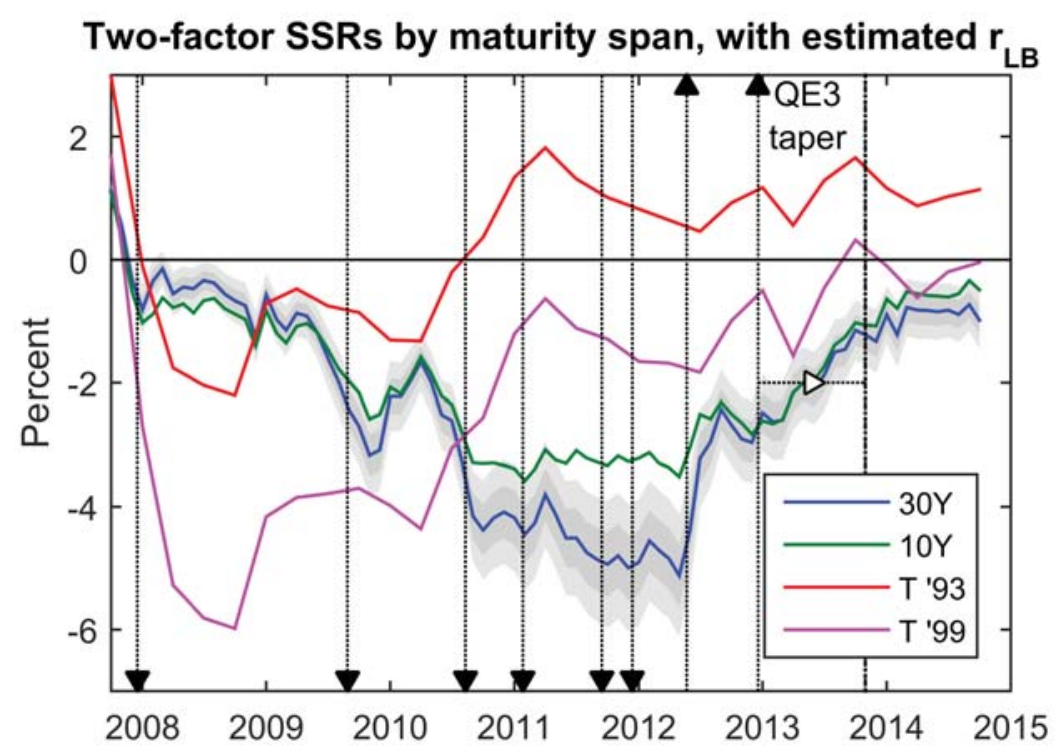

Figure 14: SSRs from K-ANSM(2) estimated with data to September 2015 and using different maturity spans. The profiles and dynamics of the SSRs are very similar, but the magnitudes have some sensitivity. The arrows indicate major unconventional policy events, which are listed and discussed in section 3.3.

As illustrated in figure 14, the profiles and dynamics of the 30-year K-ANSM(2) SSR estimates are very similar to the 10-year SSR estimates. Over the LB period, the correlations with the 10-year SSR estimates from the previous section and 30-year SSR estimates are greater than 0.95 , and the results of Kendall correlations between SSR changes and major monetary policy events are very similar to those reported in table 2 . However, the extended maturity span introduces some further magnitude sensitivity, with the 30-year

\footnotetext{
${ }^{34}$ I have also obtained SSR estimates using the calibrated LB parameters in section 5.2, which have profiles and dynamics very similar to the results presented in figure 14. The magnitude sensitivities between different LB specifications are also very similar those already documented in section 5.2, i.e. a range of -6 percent to -4 percent for the minimum SSR estimates obtained with LB parameters of 25 and zero bps.

${ }^{35}$ However, Krippner (2015c) and the website results use OIS data from January 2006, because OIS relate directly to FFR expectations (given OIS securities are settled on future FFR realizations). Krippner $(2015 \mathrm{c})$ and the website results also use a heteroskedastic specification for measurement errors, and footnote 29 explains why it is better to use a homoskedastic specification. That change will be made in due course. It makes little difference to the profile and magnitudes of the results, and the improvement is that the SSR estimates become less volatile.
} 
K-ANSM(2) SSR estimates reaching lows of around $-5 \%$, compared to around $-3.5 \%$ for the 10-year K-ANSM(2). These results confirm those reported in Krippner (2013b) with respect to maturity span.

Relating to the subsequent discussion in section 5.4, figure 14 also includes two standard Taylor rule rates (Taylor 1993, 1999), to provide an order of magnitude comparison for the SSR estimates. It is not possible to make a formal comparison, because Krippner (2013c) discusses why negative Taylor rule rates are not in principle directly comparable to negative SSRs. However, if one had a preferred Taylor rule for policy monitoring and quantitative analysis in the non-LB period, then the order of magnitude provided by that Taylor rule in the LB period could be used as a calibration mechanism for selecting a preferred SSR series. On that basis, for example, if one preferred the Taylor (1999) rule rate, then the 30-year SSR series in figure 14 would be preferable to the 10-year SSR series.

Independently from any Taylor rule considerations, the 30-year SSR series presented in figure 14 above applies the least judgement. In particular, it uses an estimated LB parameter (which avoids any need to justify a particular calibrated value and simply accepts what the yield curve data delivers), and it also uses the benchmark maturity interest rates for the entire span of available yield curve data (which avoids excluding any data).

\subsection{Discussion}

The profile and dynamics properties discussed in the previous two sections suggest that K-ANSM(2) SSR estimates will at least be useful as an ordinal measure of the stance of monetary policy, and will therefore be applicable for monitoring the stance of monetary policy. That is, for any K-ANSM(2), decreases in the SSR in response to unconventional monetary policy events indicate a market perception of more accommodative policy, and increases indicate a market perception of more restrictive policy. In that capacity, appendix A shows that K-ANSM(2) SSR estimates would play the same role as "policy lift-off" metrics.

Regarding quantitative analysis, which depends on the cardinal results for $\operatorname{K}-\operatorname{ANSM}(2)$ SSRs that are used as data, the implications are more nuanced. On the face of it, the similar profiles and dynamics of $\mathrm{K}-\operatorname{ANSM}(2)$ SSR estimates obtained with different LB parameters and maturity spans suggests that the results of any quantitative analysis may not be overly sensitive. However, the magnitudes of negative SSRs relative to positive SSRs (or relative to observed policy/short-maturity rates, given the latter are always closely replicated by the positive SSR estimates in the non-LB period) will necessarily change between different $\mathrm{K}-\operatorname{ANSM}(2)$ applications. This issue may be important depending on the nature of the quantitative analysis.

Therefore, if a K-ANSM(2) SSR series is used as a single monetary policy metric for quantitative analysis over periods spanning non-LB and LB periods, it will be important to test the robustness of those results with a variety of alternative estimates. This includes SSRs obtained from SLMs using an appropriate range of LB parameters, with the latter informed by relevant market data and the monetary policy mechanism for the given economy, and also yield curve data of different maturity spans. As mentioned in section 5.3, arguably SSR estimates from the K-ANSM(2) with an estimated LB parameter and using yield curve data out to 30 years is a natural benchmark, because it applies the least 
judgement. The conclusion and the appendix also discuss the importance of robustness testing with alternative metrics for the stance of monetary policy.

A complement to the robustness testing in the previous paragraph would be to use information external to the yield curve data to justify a particular K-ANSM(2) SSR series or to appropriately scale the negative SSRs of a K-ANSM(2) SSR series. For example, the discussion in section 5.3 on the Taylor (1999) rule suggests it could be used to justify minimum SSR estimates in the order of -5 percent (or a calibration based on the integral of the negative Taylor rule rates versus the negative SSR estimates may be more appropriate). Scaling negative SSRs using non-LB period relationships between financial market variables (e.g. equity market prices and exchange rates) and policy/short maturity is another potential means of calibration.

Note that if K-ANSM(2) SSR estimates are used as a single monetary policy metric over just the LB period, or with an appropriate allowance for a structural break between non-LB and LB periods, then the similar profiles and dynamics between different SSR estimates indicates that the results of any quantitative analysis are likely to be substantially the same. That is, the magnitudes of the levels and changes of different SSR estimates will vary over the LB period, but the estimated parameters of the quantitative model for the LB period will scale accordingly. Again, in that capacity K-ANSM(2) SSR estimates would play the same role as "policy lift-off" metrics, as discussed in appendix A.

Interestingly, if any of the K-ANSM(2) SSR estimates were used in the WX FAVAR, they would likely produce a greater effect from unconventional monetary policy than the WX results. Specifically, the more negative and persistent values of the K-ANSM(2) SSR estimates suggest that the monetary stimulus from unconventional monetary policy in the United States was larger than indicated by the WX SSR estimates, and this would likely have been responsible for a greater reduction in the unemployment rate.

As a final point for this section, two-factor SLMs will obviously fit the yield curve data less closely than three-factor SLMs, but this seems to be the trade-off required for obtaining relatively robust SSR estimates from a straightforward application of SLMs to yield curve data. Indeed, Ichiue and Ueno (2013) also suggests using SLMs with less than three factors to avoid over-fitting and counterintuitive SSR estimates:

Another reason for focusing on two-factor models is that when interest rates are stuck at the ZLB and do not move much, a large part of the information required to identify the factors is missing, and thus the number of factors may have to be smaller than that when interest rates are far from zero. ... Thus, this [Christensen and Rudebusch (2015a)] result suggests that threefactor [Shadow Rate Models] are likely to over-fit the data and to produce unrealistic estimates.

The last sentence is in reference to the counterintuitive and materially persistent positive SSR estimates obtained from the Christensen and Rudebusch (2015a) three-factor SLM application for Japan after 2008. Conversely, the SSR estimates for the Christensen and Rudebusch (2015a) one- and two-factor SLMs are broadly consistent with the evolution of conventional and unconventional monetary policy for Japan over the Christensen and Rudebusch (2015a) sample period from the mid-1990s, and similar Japanese SSR results are reported in Kim and Singleton (2012) using a two-factor SLM.

If a closer fit to the data is required for a particular application, then it is perfectly valid to use a three-factor SLM, but the resulting SSRs are not robust and should not be 
used. As discussed at the end of section 4.2, imposing a suitable set of constraints may obtain relatively robust SSRs and a close fit to the data if both elements are required. For example, one very straightforward hybrid model could use the two-factor SLM for an initial estimation (a constraint to obtain a relatively robust SSR estimate), and then fit a third factor to the resulting residuals (allowing flexibility to obtain closer fit to the data, as typically obtained with three-factor models).

\section{Conclusion}

In this article, I have shown that the dynamics and profiles of SSR estimates from threefactor SLMs are very sensitive. For example, using slightly different LB parameters and/or reestimating with updated samples of yield curve data obtains SSR estimates with very different profiles and dynamics than those presented in WX and updated monthly. Such results hold in general, and so three-factor SSRs should therefore not be used for monitoring the levels and changes in the stance of unconventional monetary policy, or for quantitative analysis.

However, I show that SSR estimates from two-factor SLMs are relatively robust, in dynamics and profile, and correlate well with unconventional monetary policy events. As such, two-factor SSRs appear to be suitable candidates for policy monitoring and quantitative analysis but, depending on the application, robustness checks with different LB specifications and samples appropriate to the given economy of application should be employed.

The robust checks should ideally also include alternative monetary policy metrics, because SSRs are unlikely to be a complete summary metric for the stance of monetary policy. ${ }^{36}$ Several such alternative metrics can be obtained from SLMs (and also see footnote 1 for a non-SLM metric). First are "policy rate lift-off" metrics, which are more robust to model specification and data than SSRs, although they are only available in unconventional periods. However, in turns out that the information inherent in lift-off metrics from two- or three-factor SLMs is already well-replicated by the negative SSRs obtained from two-factor SLMs. Appendix A contains further discussion related to these points.

Another SLM metric is the Effective Monetary Stimulus as outlined in Krippner (2015c). This metric is more comprehensive in principle than either SSRs or lift-off metrics, because it allows for the entire path of expected lower-bounded short rates relative to an estimated long-horizon natural rate. However, estimating or calibrating a long-horizon natural rate involves model extensions and additional data, and the results will remain subject to model and estimation uncertainties that need to be investigated and accounted for in practice.

Moving beyond univariate summary metrics, one can estimate expected policy and risk premium components from SLMs. This decomposition provides a very comprehensive basis for policy monitoring and quantitative analysis, particularly given that expected

\footnotetext{
${ }^{36}$ Indeed, the FFR is not necessarily a complete metric in conventional monetary policy periods. Theoretically, standard monetary theory emphasizes the role of policy expectations for monetary policy transmission, e.g. see Walsh (2003). Empirically, Gürkaynak, Sack, and Swanson (2005) is one example establishing that FOMC statements affect financial markets via surprises to both the FFR target and the expected path of the FFR target (even if no change to the FFR target occurs).
} 
policy and risk premium components respectively correspond to the channels of policy rate guidance and portfolio balance that are considered key for unconventional monetary policy. ${ }^{37}$ However, obtaining sufficient precision for the decomposition again requires model extensions and additional data, ${ }^{38}$ and the comments above on model and estimation uncertainties again apply.

In summary, the range of potential indicators for the stance of monetary policy when policy rates are constrained by the lower-bound is still in development, and their empirical applicability is still being tested. Unless or until a leading candidate is obtained, SSR estimates obtained from two-factor SLMs, while certainly not perfect in all respects, have many favorable properties and deserve to retain a place in the suite of unconventional monetary policy indicators.

\section{References}

Bauer, M. and C. Neely (2014). International channels of the FedŠs unconventional monetary policy. Journal of International Money and Finance 44, 24-46.

Bauer, M. and G. Rudebusch (2015). Monetary policy expectations at the zero lower bound. Working Paper, Federal Reserve Bank of San Francisco 18.

Black, F. (1995). Interest rates as options. Journal of Finance 50(7), 1371-1376.

Bomfim, A. (2003). 'Interest Rates as Options': assessing the markets' view of the liquidity trap. Working Paper, Federal Reserve Board of Governors 45.

Bullard, J. (2012). Shadow Interest Rates and the Stance of U.S. Monetary Policy. Speech at the Annual Conference, Olin Business School, Washington University in St. Louis, 8 November 2012.

URL: http://www.stlouisfed.org/newsroom/displayNews.cfm?article=1574.

Bullard, J. (2013). Perspectives on the Current Stance of Monetary Policy. Speech at the NYU Stern, Center for Global Economy and Business, 21 February 2013. URL: http://www.prweb.com/releases/2013/2/prweb10455633.htm.

Christensen, J., F. Diebold, and G. Rudebusch (2011). The affine arbitrage-free class of Nelson-Siegel term structure models. Journal of Econometrics 164(1), 4-20.

Christensen, J. and G. Rudebusch (2014). Estimating shadow-rate term structure models with near-zero yields. Working Paper, Federal Reserve Bank of San Francisco 39.

Christensen, J. and G. Rudebusch (2015a). Estimating shadow-rate term structure models with near-zero yields. Journal of Financial Econometrics 13(2), 226-259.

Christensen, J. and G. Rudebusch (2015b). A probability-based stress test of Federal Reserve assets and income. Journal of Monetary Economics (forthcoming).

Francis, N., L. Jackson, and M. Owyang (2014). How has empirical monetary policy analysis changed after the financial crisis? Working Paper, Federal Reserve Bank of St. Louis 2014-19A.

\footnotetext{
${ }^{37}$ See Woodford (2012) for general discussion, and Bauer and Neely (2014) for a related application of expected policy and risk premium components from a term structure model (albeit without a lower bound mechanism) to investigate the relative strength of both channels.

${ }^{38}$ For example, interest rate survey data as in Kim and Orphanides (2012), and/or macroeconomic data.
} 
Gorovoi, V. and V. Linetsky (2004). Black's model of interest rates as options, eigenfunction expansions and Japanese interest rates. Mathematical Finance 14(1), 4978.

Gürkaynak, R., B. Sack, and E. Swanson (2005). Do actions speak louder than words? The response of asset prices to monetary policy actions and statements. International Journal of Central Banking 1(1), 55-93.

Gürkaynak, R., B. Sack, and J. Wright (2007). The U.S. Treasury yield curve: 1961 to the present. Journal of Monetary Economics 54(8), 2291-2304.

Hamilton, J. (2013, 10 November). Summarizing monetary policy. URL: http://www.econbrowser.com /archives/2013/11/summarizing-mon.html.

Higgins, P. and B. Meyer (2013, 20 November). The Shadow Knows (the Fed Funds Rate). URL: http://macroblog.typepad.com/macroblog/2013/11/theshadow-knows-the-fed-funds-rate.html.

Ichiue, H. and Y. Ueno (2006). Monetary policy and the yield curve at zero interest: the macro-finance model of interest rates as options. Working Paper, Bank of Japan 06E-16.

Ichiue, H. and Y. Ueno (2013). Estimating term premia at the zero lower bound: an analysis of Japanese, US, and UK yields. Working Paper, Bank of Japan 13-E-8.

Jarrow, R. (2013). The zero-lower bound on interest rates: Myth or reality? Finance Research Letters 10(4), 151-156.

Kim, D. and A. Orphanides (2012). Term structure estimation with survey data of interest rate forecasts. Journal of Financial and Quantitative Analysis 47(1), 241272.

Kim, D. and M. Priebsch (2013). Estimation of multi-factor shadow rate term structure models. Preliminary Draft.

Kim, D. and K. Singleton (2012). Term structure models and the zero bound: an empirical investigation of Japanese yields. Journal of Econometrics 170(1), 32-49.

Krippner, L. (2006). A theoretically consistent version of the Nelson and Siegel class of yield curve models. Applied Mathematical Finance 13(1), 39-59.

Krippner, L. (2011). Modifying Gaussian term structure models when interest rates are near the zero lower bound. Discussion paper, Centre for Applied Macroeconomic Analysis 36/2011.

Krippner, L. (2013a). Measuring the stance of monetary policy in zero lower bound environments. Economics Letters 118(1), 135-138.

Krippner, L. (2013b). A tractable framework for zero-lower-bound Gaussian term structure models. Discussion Paper, Reserve Bank of New Zealand DP2013/02.

Krippner, L. (2015a). Monetary policy measures from shadow/lower bound term structure models. Presentation from 26 August 2015 ECB workshop.

Krippner, L. (2015b). A theoretical foundation for the Nelson-Siegel class of yield curve models. Journal of Applied Econometrics 30, 97-118.

Krippner, L. (2015c). Zero Lower Bound Term Structure Modeling: A Practitioner's Guide. Palgrave-Macmillan. 
Lemke, W. and A. Vladu (2015). A shadow-rate term structure model for the euro area. Presentation and draft paper from 26 August 2015 ECB workshop.

Lombardi, M. and F. Zhu (2014). A shadow policy rate to calibrate US monetary policy at the zero lower bound. Working Paper, Bank of International Settlements 452.

Nelson, C. and A. Siegel (1987). Parsimonious modelling of yield curves. Journal of Business 60(4), 473-489.

Priebsch, M. (2013). Computing arbitrage-free yields in multi-factor Gaussian shadowrate term structure models. Working Paper, Federal Reserve Board 2013-63.

Taylor, J. (1993). Discretion versus policy rules in practice. Carnegie-Rochester Conference Series on Public Policy 39, 195-214.

Taylor, J. (1999). A historical analysis of monetary policy rules. In J. Taylor (Ed.), Monetary Policy Rules, pp. 319-341. Chicago: University of Chicago.

Walsh, C. (2003). Monetary Theory and Policy, Second Edition. MIT Press.

Woodford, M. (2012). Methods of policy accommodation at the interest-rate lower bound. Speech at Jackson Hole Symposium, 20 August 2012. URL: www.kc.frb.org/publicat/sympos/2012/mw.pdf.

Wu, J. and F. Xia (2015). Measuring the macroeconomic impact of monetary policy at the zero lower bound. Journal of Money, Credit and Banking (forthcoming) 2015.

Zumbrun, J. (2014, 1 February). Bernanke secret sauce drops Fed rate as QE quantified. URL: http://www.bloomberg.com/news/2014-01-31/bernanke-secret-saucedrops-effective-fed-rate-as-qe-quantified.html.

\section{A Liftoff and the information in two factor SSRs}

In this appendix, I establish that $\mathrm{K}-\operatorname{ANSM}(2)$ SSRs provide very similar information to "policy lift-off" metrics that have been proposed as an alternative summary monetary policy metric obtainable from SLMs.

WX presents what I will call the Lift-off Horizon, which is the median time to a threshold of 25 bps from a sample of simulations of the future shadow rate path. Bauer and Rudebusch (2015) also proposes the same metric. Krippner (2015c) proposes the Expected Time to Zero (ETZ), which is the horizon at which the expected path of the SSR intercepts zero (or any other threshold may be used, but I will use zero below). Bauer and Rudebusch (2015) also proposes the equivalent metric (with a threshold of 25 bps) in the guise of a modal lift-off horizon, referencing its use in Kim and Singleton (2012), and shows that the Lift-off Horizon and ETZ metrics are very similar.

All of the authors above show empirically that lift-off metrics are less sensitive than SSRs to model specification, including whether using two or three factors and/or alternative LB parameters. Figures 12 and 13 below also confirm that finding. Hence, lift-off metrics suggest themselves as a robust monetary policy metric relative to SSRs, although I will provide one caveat at the end of this section.

However, it turns out that the information contained in lift-off metrics, whether from two- or three-factor SLMs, can summarized almost equivalently by a series of negative K-ANSM(2) SSRs. To illustrate this empirically for lift-off metrics from two-factor SLMs, 
figure 12 plots the ETZs obtained from the K-ANSM(2) specifications detailed in section 5.2 along with the SSR for the $\mathrm{K}-\operatorname{ANSM}(2)$ with an estimated LB parameter (or any other K-ANSM(2) series could be used, given their similar profiles and dynamics; ). The profiles and dynamics correspond well, and the correlations between the ETZs and the SSR are all greater than 0.94 .

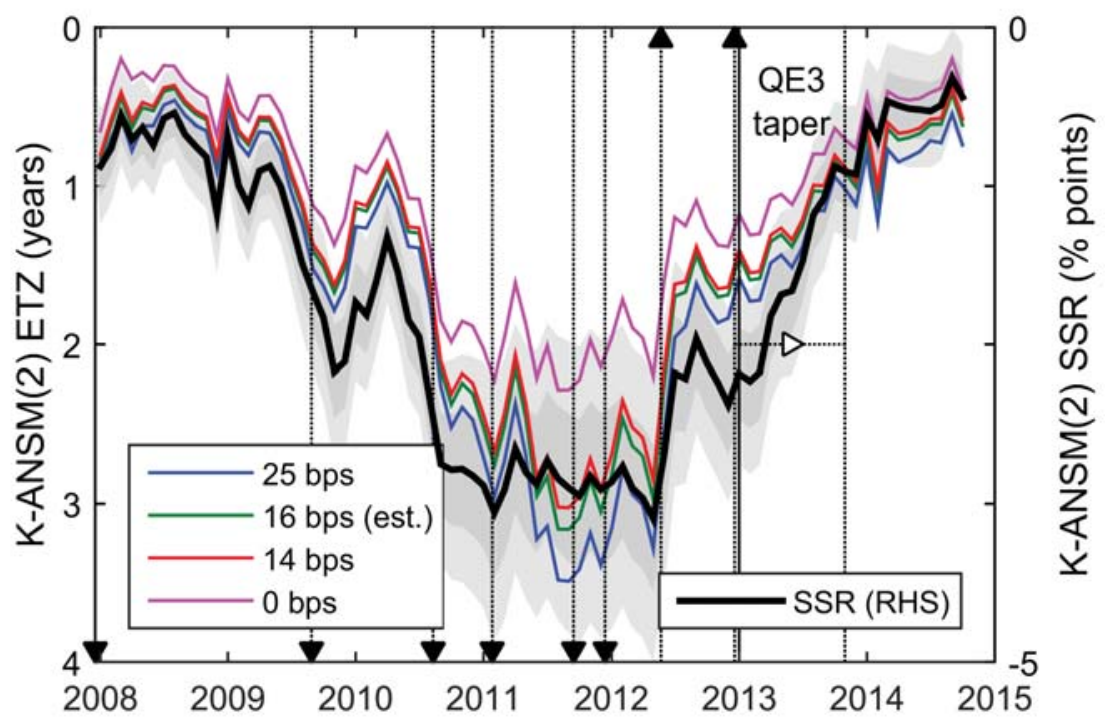

Figure 15: ETZs from K-ANSM(2) estimated with data to December 2013 (with a 10-year maturity span) and using different LB parameters. The magnitudes, profiles, and dynamics of the ETZs are very similar, and also correlate closely to the K-ANSM(2) SSR series. The arrows indicate major unconventional policy events, which are listed and discussed in section 3.3.

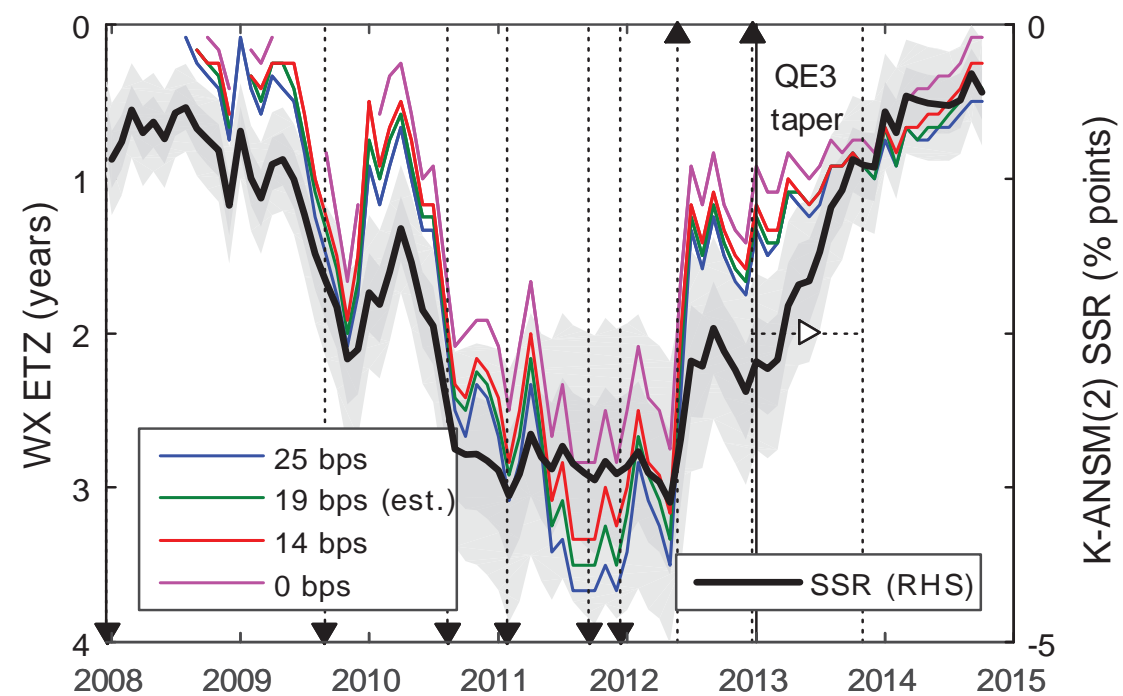

Figure 16: Figure 15: ETZs from WX model estimated with data to December 2013 and using different LB parameters. The magnitudes, profiles, and dynamics of the ETZs are very similar, and also correlate well with the K-ANSM(2) SSR series. The arrows indicate major unconventional policy events, which are listed and discussed in section 3.3.

To illustrate the analogous case empirically for three-factor liftoff metrics, figure 13 plots the ETZs I have obtained from the WX model specifications in section 3.1 along 
with the SSR for the K-ANSM(2) with an estimated LB parameter. ${ }^{39}$ The profiles and dynamics again correspond well. The correlations between the ETZ estimates, excluding any undefined values, and the representative SSR over the sample period are all greater than 0.90 .

The ETZ results above, like the WX Lift-off Horizon results, are all under the riskadjusted $\mathbb{Q}$ measure. Krippner (2015a) shows that ETZs under the physical $\mathbb{P}$ measure also have profiles and dynamics that correspond closely and are highly correlated with K$\operatorname{ANSM}(2)$ SSRs. ${ }^{40}$ The modal lift-off measures reported in Bauer and Rudebusch (2015) under both the $\mathbb{Q}$ and $\mathbb{P}$ measures also have similar profiles and dynamics to the $\mathrm{K}$ $\operatorname{ANSM}(2) \mathrm{SSR}$ in figures 15 and 16.

It can be shown that the close relationship between two-factor SSRs and two- and three-factor lift-off horizons illustrated above are general, rather than being empirical coincidences. Specifically, the expected path of the SSR for the K-ANSM(2) is:

$$
\tilde{\mathbb{E}}_{t}\left[\mathrm{r}\left(x_{t}, t+\tau\right)\right]=L_{t}+S_{t} \cdot \exp (-\tilde{\phi} \tau)
$$

and setting $\tilde{\mathbb{E}}_{t}\left[\mathrm{r}\left(x_{t}, t+\tau\right)\right]$ equal to an arbitrary threshold of $\mathrm{r}^{*}$ and re-arranging gives the ETZ result:

$$
\tau_{L}=\frac{1}{\tilde{\phi}} \log \left[\frac{S_{t}}{\mathrm{r}^{*}-L_{t}}\right]
$$

Given the SSR $\mathrm{r}_{t}=L_{t}+S_{t}$, when $\mathrm{r}_{t}$ is negative $S_{t}=-\left|\mathrm{r}_{t}\right|-L_{t}$. Substituting the latter into equation 13 gives:

$$
\tau_{L}=\frac{1}{\tilde{\phi}} \log \left[\frac{L_{t}+\left|\mathrm{r}_{t}\right|}{L_{t}-\mathrm{r}^{*}}\right]
$$

The Level state variable $L_{t}$ is relatively non-cyclical compared to $\left|\mathrm{r}_{t}\right|$. Hence, movements in $\left|\mathrm{r}_{t}\right|$ when $\mathrm{r}_{t}$ is negative will be approximately monotonic with movements in $\tau_{L}$, thus explaining the correlation between the two-factor empirical results discussed above. This result can be seen even more clearly for the case when $L_{t}>\left|\mathrm{r}_{t}\right| \gg \mathrm{r}^{*}$, hence $L_{t}-\mathrm{r}^{*} \simeq L_{t}$ and the first-order Taylor expansion of $\log [1+x] \simeq x$ gives:

$$
\tau_{L}=\frac{1}{\tilde{\phi}} \log \left[1+\frac{\left|\mathrm{r}_{t}\right|}{L_{t}}\right] \simeq \frac{1}{\tilde{\phi}} \frac{\left|\mathrm{r}_{t}\right|}{L_{t}}
$$

although higher-order terms become important when $L_{t} \lesssim\left|\mathrm{r}_{t}\right|$.

Regarding three-factor ETZ results versus the two-factor K-ANSM(2), Krippner (2015c) has already shown empirically that the ETZ estimates from K-ANSM(2) and K-ANSM(3) specifications are very close. The following line of reasoning explains the principles behind those empirical results: (1) the expected path of the SSR $\tilde{\mathbb{E}}_{t}\left[\mathrm{r}\left(x_{t}, t+\tau\right)\right]$ for longer horizons $\tau$ is relatively insensitive to model specifications; (2) the relative insensitivity occurs

\footnotetext{
${ }^{39} \mathrm{ETZ}$ estimates are sometimes undefined due to the entire expected path of the WX SSR being above zero. Note also that I follow Krippner (2015c) in using the largest of the zero intercepts if there are more than one (which can occur due to the non-monotonicity of shadow yield curve in three-factor SLMs). This choice obtains the intercept going up through zero, whereas the first intercept would be going down through zero due to counterintuitive positive SSRs at short horizons.

${ }^{40}$ Lift-off metrics under the physical $\mathbb{P}$ measure are more relevant than $\mathbb{Q}$-measure lift-off metrics because they relate directly to the "real world" or actual expectations held by market participants, including those responsible for surveys. $\mathbb{Q}$-measure lift-off metrics relate to the "risk-adjusted world", and will differ from $\mathbb{P}$-measure lift-off metrics depending on the risk premium.
} 
because the estimated SLM results for longer horizons are more beholden to the data; and (3) the SLM is more beholden to the data because the lower-bound factor loadings are only partially attenuated with respect to the shadow factor loadings (see figures 6,8 , and 13). Hence, given that K-ANSM(2) SSRs correlate closely to K-ANSM(2) ETZs, and the latter relate closely to K-ANSM(3) ETZs, K-ANSM(2) SSRs will therefore correlate closely to K-ANSM(3) ETZs.

The caveat when using lift-off metrics for quantitative analysis is that they are only available during unconventional periods. This will not present an issue for policy monitoring. However, lift-off metrics will not be usable as standalone series for quantitative analysis that spans both conventional and unconventional periods. It may be possible to incorporate lift-off metrics as a type of dummy variable in combination with the FFR, but the discussion above shows that using the time series of negative SSR estimates would provide the same inherent information. Alternatively, using the full SSR series with both negative and positive values avoids any need to somehow combine two distinct monetary policy metrics within any quantitative analysis, but the robustness checks mentioned in section 5.3 will be necessary to ensure that the magnitude sensitivity of negative SSR estimates is not unduly affecting the results of the analysis. 